\title{
On Topological Indices of Total Graph and Its Line Graph for Kragujevac Tree Networks
}

\author{
Salma Kanwal, ${ }^{1}$ Ayesha Riasat, ${ }^{2}$ Muhammad Kamran Siddiqui $\left(\mathbb{D},{ }^{3}\right.$ Shabnam Malik, ${ }^{4}$ \\ Khadija Sarwar, ${ }^{1}$ Afzal Ammara, ${ }^{1}$ and Asare-Tuah Anton ${ }^{5}{ }^{5}$ \\ ${ }^{1}$ Department of Mathematics, Lahore College for Women University, Lahore, Pakistan \\ ${ }^{2}$ University of Engineering and Technology, KSK Campus, Lahore, Pakistan \\ ${ }^{3}$ Department of Mathematics, COMSATS University Islamabad, Lahore Campus, Lahore, Pakistan \\ ${ }^{4}$ Forman Christian College(A Chatered University), Lahore, Pakistan \\ ${ }^{5}$ Department of Mathematics, University of Ghana, Legon, Ghana \\ Correspondence should be addressed to Asare-Tuah Anton; aasare-tuah@ug.edu.gh
}

Received 16 September 2021; Accepted 30 October 2021; Published 18 November 2021

Academic Editor: Baltazar Aguirre Hernandez

Copyright (c) 2021 Salma Kanwal et al. This is an open access article distributed under the Creative Commons Attribution License, which permits unrestricted use, distribution, and reproduction in any medium, provided the original work is properly cited.

Kragujevac tree is indicated by $K ; K \in K g_{q=s(2 t+1)+1, s}$ with order and size $s(2 t+1)+1$ and $s(2 t+1)$, respectively. In this paper, we have a look at certain topological features of the total graph and line graph of the total graph of the considered tree, i.e., Kragujevac tree, by computing different topological indices and polynomials.

\section{Introduction}

The graphs throughout the discussion are simple. In a graph $H, V(H)$ and $E(H)$ denote the sets of nodes and lines accordingly. Consider $d_{H}(u)$ be the degree of a vertex $u$. Here, we assume that $A_{1}, A_{2}, A_{3}, \ldots$ are the branches which are shown in Figure 1. A Kragujevac tree [1-3] with central vertex consisting of degree at least 3 is a proper tree having branches $A_{1}, A_{2}, A_{3}, \ldots$.

A group of proper Kragujevac trees whose central vertex consist of degree $s$ and order $q$ is symbolized by $K g_{q, s}$, and by adding a new vertex of degree 2 to a pendant line, an improper Kragujevac tree is attained [4]. It is symbolized by $K g_{q, s}$; see [5-7] for more details.

Here, we have the branches $A_{l_{1}}, A_{l_{2}}, \ldots, A_{l_{m}}$ where $l_{i} \geq 2$ for all $\{i=1, \ldots, m\}$ connected to the mid vertex. Our concern in this research work is with a specific type of Kragujevac tree when $A_{l_{1}}, A_{l_{2}}, \ldots, A_{l_{m}}$ are identical, i.e., $l_{1}=l_{2}=\cdots=l_{m}=p$. Thus, order and size of Kragujevac tree $K \in K g_{q, s}$ are $q=s(2 t+1)+1$ and $s(2 t+1)$ accordingly, see Figure 2.
Any function on a graph that does not rely on the labelling of its vertices is referred to as a graph invariant or a topological index. Many invariants have been used in QSPR/QSAR studies with varying degrees of success. Gutman and Trinajstic [8] proposed Zagreb indices three decades before. Balaban et.al. later referred to them as Zagreb group indices $[9,10]$.

Then, the first Zagreb index and second Zagreb index are formulated as

$$
\begin{aligned}
& M_{1}(H)=\sum_{l \in V(H)}\left(d_{l}\right)^{2}=\sum_{m l \in E(H)} d_{m}+d_{l}, \\
& M_{2}(H)=\sum_{m l \in E(H)} d_{m} d_{l} .
\end{aligned}
$$

In 2011, Fath-Tabar [11] suggested third Zagreb index for some graph $H$ symbolized by $M_{3}(H)$ and is specifically defined as

$$
M_{3}(H)=\sum_{m l \in E(H)}\left|d_{m}-d_{l}\right| .
$$




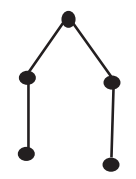

$\mathrm{A}_{2}$

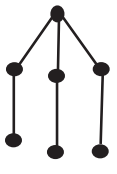

$\mathrm{A}_{3}$

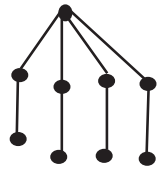

$\mathrm{A}_{4}$

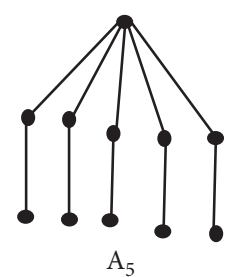

$\mathrm{A}_{5}$

Figure 1: Branches (a) $A_{2}$, (b) $A_{3}$, (c) $A_{4}$, and (d) $A_{5}$.

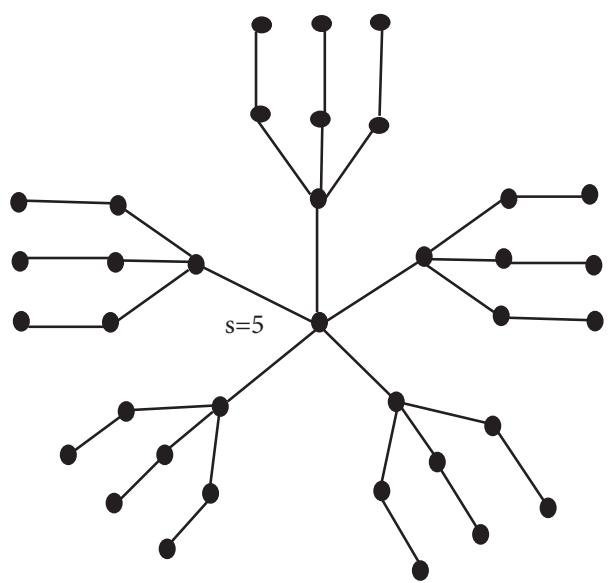

Figure 2: Kragujevac tree $K$ from class $K g_{36,5}$.

The same author introduced Zagreb polynomials in the same year, naming them first, second, and third Zagreb polynomials, and they are formulated as

$$
\begin{aligned}
& M_{1}(H, u)=\sum_{m l \in E(H)} u^{d_{m}+d_{l}}, \\
& M_{2}(H, u)=\sum_{m l \in E(H)} u^{d_{m} d_{l}}, \\
& M_{3}(H, u)=\sum_{m l \in E(H)} u^{\left|d_{m}-d_{l}\right|},
\end{aligned}
$$

respectively. Modified Zagreb indices [12] are another variant of Zagreb indices that are associated with degrees of vertices. The modified Zagreb indices first and second are signified by $m M_{1}(H)$ and $m M_{2}(H)$, respectively, and are defined as

$$
\begin{aligned}
& { }^{m} M_{1}(H)=\sum_{m l \in E(H)} \frac{1}{d_{m}+d_{l}}, \\
& { }^{m} M_{2}(H)=\sum_{m l \in E(H)} \frac{1}{d_{m} d_{l}} .
\end{aligned}
$$

Hyper-Zagreb index, proposed by Shirdel et.al. [13], was formulated as

$$
H M(H)=\sum_{m l \in E(H)}\left(d_{m}+d_{l}\right)^{2} .
$$

In 2013, Ranjini et.al. suggested redefined Zagreb indices [14] and were formulated as

$$
\begin{aligned}
\text { redefined first Zagreb index } & =\operatorname{Re} M_{1}(H)=\sum_{m l \in E(H)} \frac{d_{m}+d_{l}}{d_{m} d_{l}}, \\
\text { redefined second Zagreb index } & =\operatorname{Re} M_{2}(H)=\sum_{m l \in E(H)} \frac{d_{m} d_{l}}{d_{m}+d_{l}}, \\
\text { redefined third Zagreb index } & =\operatorname{Re} M_{3}(H)=\sum_{m l \in E(H)}\left(d_{m} d_{l}\right)\left(d_{m}+d_{l}\right) .
\end{aligned}
$$


Furtula et.al. introduced forgotten index which is marked by $F(H)$ for graph $H$ [15], formulated as

$$
F(H)=\sum_{l \in V(H)}\left(d_{l}\right)^{3}=\sum_{m l \in E(H)}\left[\left(d_{m}\right)^{2}+\left(d_{l}\right)^{2}\right] .
$$

Parallel to the notion of forgotten topological index is that of forgotten polynomial. Forgotten polynomial of a graph $H$ is given by

$$
F(H, u)=\sum_{m l \in E(H)} u^{\left[\left(d_{m}\right)^{2}+\left(d_{l}\right)^{2}\right]} .
$$

Reciprocal Randić index, reduced second Zagreb index, and reduced reciprocal Randić index were proposed by Gutman et al. [16] in 2014 as follows:

$$
\begin{aligned}
\operatorname{RR}(H) & =\sum_{m l \in E(H)} \sqrt{\left(d_{m}\right)\left(d_{l}\right)}, \\
R M_{2}(H) & =\sum_{m l \in E(H)}\left(d_{m}-1\right)\left(d_{l}-1\right), \\
\operatorname{RRR}(H) & =\sum_{m l \in E(H)} \sqrt{\left(d_{m}-1\right)\left(d_{l}-1\right)} .
\end{aligned}
$$

Many new graph invariants [17-20] known as family of Gourava indices were brought into existence by V. R. Kulli in 2017 and are described as follows:

$$
\begin{aligned}
\text { first Gourava index } & =\mathrm{G}_{1} \mathrm{O}(H)=\sum_{m l \in E(H)}\left[d_{m}+d_{l}+d_{m} d_{l}\right], \\
\text { second Gourava index } & =\mathrm{G}_{2} \mathrm{O}(H)=\sum_{m l \in E(H)}\left[\left(d_{m}+d_{l}\right)\left(d_{m} d_{l}\right)\right], \\
\text { product connectivity Gourava index } & =\operatorname{PGO}(H)=\sum_{m l \in E(H)} \frac{1}{\sqrt{\left(d_{m}+d_{l}\right)\left(d_{m} d_{l}\right)}}, \\
\text { sum connectivity Gourava index } & =\operatorname{SGO}(H)=\sum_{m l \in E(H)} \frac{m}{\sqrt{\left(d_{m}+d_{l}\right)+\left(d_{m} d_{l}\right)}}, \\
\text { first hyper - Gourava index } & =\operatorname{HGO}_{1}(H)=\sum_{m l \in E(H)}\left[\left(d_{m}+d_{l}\right)+\left(d_{m} d_{l}\right)\right]^{2}, \\
\text { second hyper - Gourava index } & =\operatorname{HGO}_{2}(H)=\sum_{m l \in E(H)}\left[\left(d_{m}+d_{l}\right)\left(d_{m} d_{l}\right)\right]^{2} .
\end{aligned}
$$

$M$-polynomial [21] is given as

$$
M(H ; u, v)=\sum_{\delta \leq s \leq p \leq \Delta} x_{s p}(H) u^{s} v^{p}
$$

where $\delta$ and $\Delta$ are respectively minimum and maximum of vertex degrees in graph $H$ and $x_{s p}$ represents the number of edges $m l \in E(H)$ such that $\left\{d_{m}, d_{l}\right\}=\{s, p\}$. We can deduce various indices from $M$-polynomial such as

$$
\begin{gathered}
\text { first Zagreb index }=M_{1}(H)=\left(D_{u}+D_{v}\right)(M(H ; u, v))_{u=v=1}, \\
\text { second Zagreb index }=M_{2}(H)=\left(D_{u} D_{v}\right)(M(H ; u, v))_{u=v=1}, \\
\text { modified second Zagreb index }={ }^{m} M_{2}(H)=\left(S_{u} S_{v}\right)(M(H ; u, v))_{u=v=1}, \\
\text { Randic index }=R_{\alpha}(H)=\left(S_{u}^{\alpha} S_{v}^{\alpha}\right)(M(H ; u, v))_{u=v=1}=\sum_{m l \in E(H)}\left(d_{m} d_{l}\right)^{\alpha}, \\
\text { inverse Randic index }=\operatorname{RR}(H)=\left(D_{u}^{\alpha} D_{v}^{\alpha}\right)(M(H ; u, v))_{u=v=1}=\sum_{m l \in E(H)} \sqrt{d_{m} d_{l}} .
\end{gathered}
$$


Symmetric division deg index

$$
\begin{aligned}
\operatorname{SDD}(H) & =\left(D_{u} S_{v}+D_{v} S_{u}\right)(M(H ; u, v))_{u=v=1}=\sum_{m l \in E(H)}\left\{\frac{\min \left(d_{m}, d_{l}\right)}{\max \left(d_{m}, d_{l}\right)}+\frac{\max \left(d_{m}, d_{l}\right)}{\min \left(d_{m}, d_{l}\right)}\right\}, \\
\text { harmonic index } & =H(H)=2 S_{u} J(M(H ; u, v))_{u=v=1}=\sum_{m l \in E(H)} \frac{2}{d_{m}+d_{l}}, \\
\text { inverse sum indeg index } & =\operatorname{ISI}(H)=S_{u} J D_{u} D_{v}(M(H ; u, v))_{u=v=1}=\sum_{m l \in E(H)} \frac{d_{m} d_{l}}{d_{m}+d_{l}},
\end{aligned}
$$

and augmented Zagreb index

$$
\begin{aligned}
A(H) & =S_{u}^{3} Q_{-2} D_{u}^{3} D_{v}^{3}(M(H ; u, v))_{u=v=1} \\
& =\sum_{m l \in E(H)}\left\{\frac{d_{m} d_{l}}{d_{m}+d_{l}-2}\right\}^{3},
\end{aligned}
$$

where

$$
\begin{aligned}
D_{u} M(H ; u, v) & =u \frac{\partial(M(H ; u, v))}{\partial u}, \\
D_{v} M(H ; u, v) & =v \frac{\partial(M(H ; u, v))}{\partial v}, \\
S_{u} M(H ; u, v) & =\int_{0}^{u} \frac{M(H ; t, v)}{t} \mathrm{~d} t, \\
S_{v} M(H ; u, v) & =\int_{0}^{v} \frac{M(H ; u, t)}{t} \mathrm{~d} t, \\
J M(H ; u, v) & =M(H ; u, u), \\
Q_{\alpha} M(H ; u, v) & =u^{\alpha} M(H ; u, v) .
\end{aligned}
$$

Since the past three years, people have conducted a lot of research on graphs, various operations, and chemical invariants of graphs. Observe simple charts under different chart operations such as line chart, subdivision, half-full point chart, half-full line chart, and general chart. The topological index of these operations on the graph is studied in depth.

Eliasi et.al. [22] displayed operations in the chart, such as $S, R, Q, T$, named subdivision, semitotal dot chart, semitotal line chart, and general chart. The subdivision graph represented by $S(H)$ is derived from $H$ by replacing each of its edges or lines with a path of length 2, or in other words, by placing a new vertex (called white vertex) on each line of $H$ [23-25]. In order to maintain the difference between the existing vertices and the newly inserted vertices, the vertices in $H$ are called black vertices. If two white vertices are adjacent to each other in $H$, it is called correlation. Similarly, if two black vertices are adjacent in $H$, it is called correlation [26, 27].

In 1981, Bertz [28] invented the first topological index established on an online graph. For these graphical operations, many authors quote $[21,29,30]$ to compute various topological indices. The general diagram of the Kragujevac tree is displayed in Figure 3.

Next part of this research paper comprises of two main Sections 2 and 3. In Section 2, we put forward computations for several chemical invariants (through direct calculations 2.2, 3.2 and using relation between polynomials 2.1, 3.1 and chemical invariants 2.3, 3.3) for Kragujevac tree. In Section 2 , graphic operation $T$, total graph is applied at $K$, whereas in Section 3, same computation is made for $L(T(K))$.

\section{Certain Topological Indices and Polynomials of $T(K)$, where $K \in K g_{q==s(2 t+1)+1, s}$}

In this section, we compute certain topological indices, polynomials, and several other chemical indices in terms of these polynomials as mentioned in previous section for the total graph of Kragujevac tree. The edge partition is depicted in Table 1.

Theorem 1. Let $H$ be the total graph of a Kragujevac tree; $K \in K g_{q=s(2 t+1)+1, s}$. Then,

$$
\begin{aligned}
F(H, u)= & \sum_{m l \in E(H)} u^{\left(d_{m}\right)^{2}+\left(d_{l}\right)^{2}}=s u^{\left[(2 s)^{2}+(s+t+1)^{2}\right]}+s u^{\left[(s+t+1)^{2}+(2 t+2)^{2}\right]} \\
& +s t u^{\left[(s+t+1)^{2}+(t+3)^{2}\right]}+\frac{s t^{2}}{2} u^{\left[(t+3)^{2}+(t+3)^{2}\right]}-\frac{s t}{2} u^{\left[(t+3)^{2}+(t+3)^{2}\right]}
\end{aligned}
$$




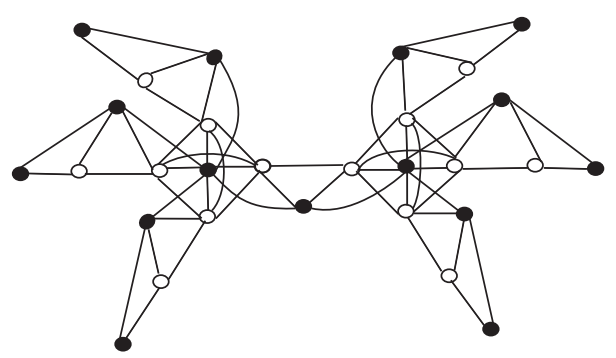

FIgURE 3: Total graph of Kragujevac tree.

TABLE 1: Line distribution in $T(K)$, where $K \in K g_{q=s(2 t+1)+1, s}$.

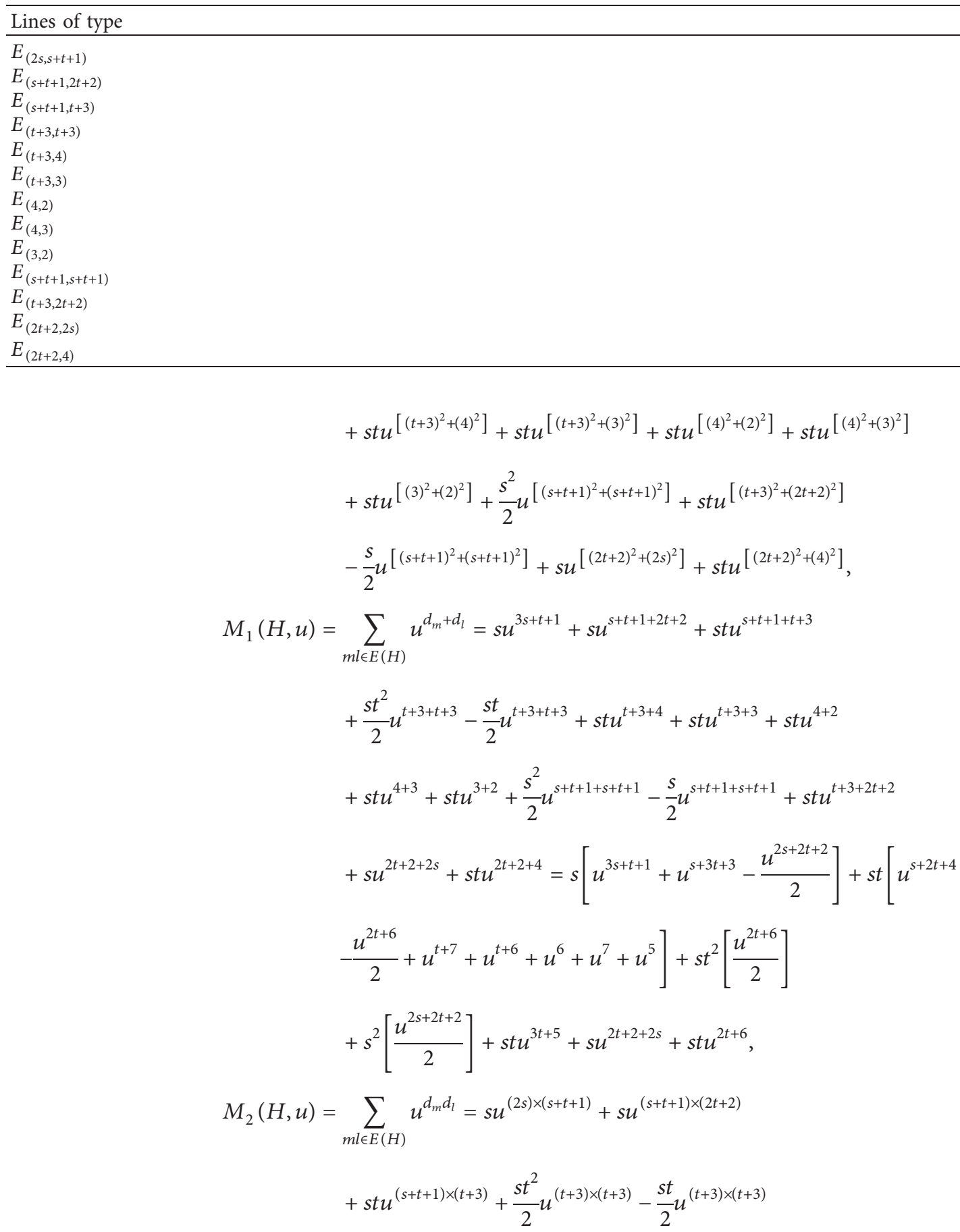

Number of lines

$s$
$s$
$s t$
$s t(t-1) / 2$
$s t$
$s t$
$s t$
$s t$
$s t$
$s(s-1) / 2$
$s t$
$s$
$s t$




$$
\begin{aligned}
& +s t u^{(t+3) \times(4)}+s t u^{(t+3) \times(3)}+s t u^{(3) \times(2)}+s u^{(2 t+2) \times(2 s)} \\
& +\frac{s^{2}}{2} u^{(s+t+1) \times(s+t+1)}-\frac{s}{2} u^{(s+t+1) \times(s+t+1)}+s t u^{(t+3) \times(2 t+2)} \\
& +s t u^{(2 t+2) \times(4)}+s t u^{(4) \times(2)}+s t u^{(4) \times(3)}+s t u_{(t+3) \times(4)}=s\left[u^{2 s^{2}+2 s t+2 s}\right. \\
& \left.+u^{2 s t+4 t+2 s+2 t^{2}+2}-\frac{u^{s^{2}+2 s t+2 s+2 t+t^{2}+1}}{2}\right]+s t\left[u^{t^{2}+s t+4 t+3 s+3}\right. \\
& \left.+\frac{u^{t^{2}+6 t+9}}{2}+u^{12}+u^{4 t+12}+u^{3 t+9}+u^{8}+u^{6}\right] \\
& +s t^{2}\left[\frac{u^{t^{2}+6 t+9}}{2}\right]+s^{2}\left[\frac{u^{s^{2}+2 s t+2 s+2 t+t^{2}+1}}{2}\right]+s t u^{2 t^{2}+8 t+6}+s u^{4 s t+4 s}+s t u^{(8 t+8)} \\
& M_{3}(H, u)=\sum_{m l \in E(H)} u^{\left|d_{m}-d_{l}\right|}=s u^{(2 s)-(s+t+1)}+s u^{(s+t+1)-(2 t+2)} \\
& +s t u^{(s+t+1)-(t+3)}+\frac{t s^{2}}{2} u^{(t+3)-(t+3)}-\frac{s t}{2} u^{(t+3)-(t+3)}+s t u^{(t+3)-(4)} \\
& +s t u^{(t+3)-(3)}+s t u^{(4)-(2)}+s t u^{(4)-(3)}+s t u^{(3)-(2)}+\frac{s^{2}}{2} u^{(s+t+1)-(s+t+1)} \\
& -\frac{s}{2} u^{(s+t+1)-(s+t+1)}+s t u^{(t+3)-(2 t+2)}+s u^{(2 t+2)-(2 s)}+s t u^{(2 t+2)-(4)}=s\left[2 u^{s-t-1}-\frac{1}{2}\right] \\
& +s t\left[u^{s-2}-\frac{1}{2}+u^{t-1}+u^{t}+u^{2}+2 u\right]+t s^{2}\left[\frac{1}{2}\right]+s^{2}\left[\frac{1}{2}\right]+s t u^{1-t}+s u^{2 t+2-2 s}+s t u^{2 t-2} \text {. }
\end{aligned}
$$

Proof. Consider the total graph of Kragujevac tree, denoted by $T(K)$, where $K \in K g_{q=s(2 t+1)+1, s}$. We categorize the lines of $T(K)$ based on the lines of type $E_{\left(d_{m}, d_{l}\right)}$, where $m l \in E(T(K))$. The line classification is shown in Table 1 ,

$$
\begin{aligned}
F(H, u)= & \sum_{m l \in E(H)} u^{\left(d_{m}\right)^{2}+\left(d_{l}\right)^{2}}=s u^{\left[(2 s)^{2}+(s+t+1)^{2}\right]}+s u^{\left[(s+t+1)^{2}+(2 t+2)^{2}\right]} \\
& +s t u^{\left[(s+t+1)^{2}+(t+3)^{2}\right]}+\frac{s t^{2}}{2} u^{\left[(t+3)^{2}+(t+3)^{2}\right]}-\frac{s t}{2} u^{\left[(t+3)^{2}+(t+3)^{2}\right]} \\
& +s t u^{\left[(t+3)^{2}+(4)^{2}\right]}+s t u^{\left[(t+3)^{2}+(3)^{2}\right]}+s t u^{\left[(4)^{2}+(2)^{2}\right]}+s t u^{\left[(4)^{2}+(3)^{2}\right]} \\
& +s t u^{\left[(3)^{2}+(2)^{2}\right]}+\frac{s^{2}}{2} u^{\left[(s+t+1)^{2}+(s+t+1)^{2}\right]}+s t u^{\left[(t+3)^{2}+(2 t+2)^{2}\right]} \\
& -\frac{s}{2} u^{\left[(s+t+1)^{2}+(s+t+1)^{2}\right]}+s u^{\left[(2 t+2)^{2}+(2 s)^{2}\right]}+s t u^{\left[(2 t+2)^{2}+(4)^{2}\right]} \\
M_{1}(H, u)= & \sum_{m l \in E(H)} u^{d_{m}+d_{l}}=s u^{3 s+t+1}+s u^{s+t+1+2 t+2}+s t u^{s+t+1+t+3} \\
& +\frac{s t^{2}}{2} u^{t+3+t+3}-\frac{s t}{2} u^{t+3+t+3}+s t u^{t+3+4}+s t u^{t+3+3}+s t u^{4+2} \\
& +s t u^{4+3}+s t u^{3+2}+\frac{s^{2}}{2} u^{s+t+1+s+t+1}-\frac{s}{2} u^{s+t+1+s+t+1}+s t u^{t+3+2 t+2}
\end{aligned}
$$




$$
\begin{aligned}
& +s u^{2 t+2+2 s}+s t u^{2 t+2+4}=s\left[u^{3 s+t+1}+u^{s+3 t+3}-\frac{u^{2 s+2 t+2}}{2}\right] \\
& +s t\left[u^{s+2 t+4}-\frac{u^{2 t+6}}{2}+u^{t+7}+u^{t+6}+u^{6}+u^{7}+u^{5}\right] \\
& +s t^{2}\left[\frac{u^{2 t+6}}{2}\right]+s^{2}\left[\frac{u^{2 s+2 t+2}}{2}\right]+s t u^{3 t+5}+s u^{2 t+2+2 s}+s t u^{2 t+6}, \\
& M_{2}(H, u)=\sum_{m l \in E(H)} u^{d_{m} d_{l}}=s u^{(2 s) \times(s+t+1)}+s u^{(s+t+1) \times(2 t+2)} \\
& +s t u^{(s+t+1) \times(t+3)}+\frac{s t^{2}}{2} u^{(t+3) \times(t+3)}-\frac{s t}{2} u^{(t+3) \times(t+3)} \\
& +s t u^{(t+3) \times(4)}+s t u^{(t+3) \times(3)}+s t u^{(3) \times(2)}+s u^{(2 t+2) \times(2 s)} \\
& +\frac{s^{2}}{2} u^{(s+t+1) \times(s+t+1)}-\frac{s}{2} u^{(s+t+1) \times(s+t+1)}+s t u^{(t+3) \times(2 t+2)} \\
& +s t u^{(2 t+2) \times(4)}+s t u^{(4) \times(2)}+s t u^{(4) \times(3)}+s t u_{(t+3) \times(4)}=s\left[u^{2 s^{2}+2 s t+2 s}\right. \\
& \left.+u^{2 s t+4 t+2 s+2 t^{2}+2}-\frac{u^{s^{2}+2 s t+2 s+2 t+t^{2}+1}}{2}\right]+s t\left[u^{t^{2}+s t+4 t+3 s+3}\right. \\
& \left.+\frac{u^{t^{2}+6 t+9}}{2}+u^{12}+u^{4 t+12}+u^{3 t+9}+u^{8}+u^{6}\right] \\
& +s t^{2}\left[\frac{u^{t^{2}+6 t+9}}{2}\right]+s^{2}\left[\frac{u^{s^{2}+2 s t+2 s+2 t+t^{2}+1}}{2}\right] \\
& +s t u^{2 t^{2}+8 t+6}+s u^{4 s t+4 s}+s t u^{(8 t+8)} \text {, } \\
& M_{3}(H, u)=\sum_{m l \in E(H)} u^{\left|d_{m}-d_{l}\right|}=s u^{(2 s)-(s+t+1)}+s u^{(s+t+1)-(2 t+2)} \\
& +s t u^{(s+t+1)-(t+3)}+\frac{t s^{2}}{2} u^{(t+3)-(t+3)}-\frac{s t}{2} u^{(t+3)-(t+3)} \\
& +s t u^{(t+3)-(4)}+s t u^{(t+3)-(3)}+s t u^{(4)-(2)} \\
& +s t u^{(4)-(3)}+s t u^{(3)-(2)}+\frac{s^{2}}{2} u^{(s+t+1)-(s+t+1)}-\frac{s}{2} u^{(s+t+1)-(s+t+1)} \\
& +s t u^{(t+3)-(2 t+2)}+s u^{(2 t+2)-(2 s)}+s t u^{(2 t+2)-(4)}=s\left[2 u^{s-t-1}-\frac{1}{2}\right] \\
& +s t\left[u^{s-2}-\frac{1}{2}+u^{t-1}+u^{t}+u^{2}+2 u\right]+t s^{2}\left[\frac{1}{2}\right]+s^{2}\left[\frac{1}{2}\right] \\
& +s t u^{1-t}+s u^{2 t+2-2 s}+s t u^{2 t-2} \text {. }
\end{aligned}
$$


Theorem 2. Let $H$ be the total graph of Kragujevac tree; $K \in K g_{q=s(2 t+1)+1, s}$, then we have

$$
\begin{aligned}
H M(H)= & 12 s^{3}+56 s t^{2}+8 s+209 s t+20 s^{2} t+10 s^{2}+5 s^{3} t+16 s t^{3}+6 s^{2} t^{2} \\
& +2 s t^{4}+2 s^{4}+13 s t^{3}+69 s t+58 s t^{2}+8 s t^{2}+4 s+4 s^{3}+8 s^{2} \\
\operatorname{Re} M_{1}(H)= & s\left[\frac{3}{2 s+2 t+2}+\frac{3}{2 s t+4 t+2 s+2 t^{2}+2}-\frac{1}{s^{2}+2 s t+2 s+2 t+t^{2}+1}\right] \\
& +s t\left[\frac{3}{2 s t+2 s+2 t^{2}+4 t+2}+\frac{2}{t^{2}+s t+4 t+3 s+3}-\frac{1}{t^{2}+6 t+9}+\frac{7}{4 t+12}+\frac{5}{6}-\frac{1}{s^{2}+2 s t+2 s+2 t+t^{2}+1}\right. \\
& +s^{2} t\left[\frac{1}{t^{2}+s t+4 t+3 s+3}+\frac{1}{s^{2}+2 s t+2 s+2 t+t^{2}+1}\right] \\
& +s t^{2}\left[\frac{2}{t^{2}+s t+4 t+3 s+3}+\frac{3}{t^{2}+6 t+9} \frac{1}{t^{2}+6 t+9}+\frac{1}{4 t+12}+\frac{1}{3 t+9}\right] \\
& +s t^{3}\left[\frac{1}{t^{2}+6 t+9}\right]+s t \frac{2 t+6}{(8 t+8)}+t\left[\frac{1}{2 t+2 s+2}\right] \\
& s^{3}\left[\frac{1}{s^{2}+2 s t+2 s+2 t+t^{2}+1}\right]+s t \frac{3 t+5}{2 t^{2}+8 t+6}+s \frac{2 t+2+2 s}{(4 s t+4 s)}, \\
& \\
& =1
\end{aligned}
$$

$\operatorname{Re} M_{2}(H)=\frac{2 s^{3}}{3 s+t+1}+\frac{2 s^{2} t}{3 s+t+1}+\frac{2 s^{2}}{3 s+t+1}+\frac{2 s^{2} t}{s+3 t+3}+\frac{4 s t}{s+3 t+3}+\frac{2 s^{2}}{s+3 t+3}+\frac{2 s t^{2}}{s+3 t+3}$$$
+\frac{2 s}{s+3 t+3}+\frac{s t^{3}}{s+2 t+4}+\frac{s^{2} t^{2}}{s+2 t+4}+\frac{4 s t^{2}}{s+2 t+4}+\frac{3 s^{2} t}{s+2 t+4}+\frac{3 s t}{s+2 t+4}+\frac{s t^{4}}{4 t+12}
$$$$
+\frac{3 s t^{3}}{2 t+6}+\frac{9 s t^{2}}{4 t+12}-\frac{s t^{3}}{4 t+12}-\frac{6 s t^{2}}{4 t+12}-\frac{9 s t}{4 t+12}+\frac{4 s t^{2}}{t+7}+\frac{12 s t}{t+7}+\frac{3 s t^{2}}{t+6}
$$$$
+\frac{9 s t}{t+6}+\frac{4 s t}{3}+\frac{12 s t}{7}+\frac{6 s t}{5}+\frac{s^{4}}{4 s+4 t+4}+\frac{s^{3} t}{2 s+2 t+2}+\frac{s^{3}}{2 s+2 t+2}+\frac{s^{2} t}{2 s+2 t+2}
$$$$
+\frac{s^{2} t^{2}}{4 s+4 t+4}+\frac{s^{2}}{4 s+4 t+4}-\frac{s^{3}}{4 s+4 t+4}-\frac{s^{2} t}{2 s+2 t+2}-\frac{s^{2}}{2 s+2 t+2}-\frac{s t}{2 s+2 t+2}
$$$$
-\frac{s t^{2}}{4 s+4 t+4}-\frac{s}{4 s+4 t+4}+s t \frac{\left(2 t^{2}+8 t+6\right)}{t+3+2 t+2}+s \frac{4 s t+4 s}{2 t+2+2 s}+s t \frac{8 t+8}{2 t+6}
$$

$\operatorname{Re} M_{3}(H)=s^{5}+s^{4}[6]+s^{3}[8]-s^{2}[8]+s[5]+36 s^{2} t+104 s t^{2}+24 s^{2} t^{2}+4 s^{2} t^{3}+13 s^{3} t$

$$
\begin{aligned}
& +42 s t^{3}+4 s^{3} t^{2}+2 s^{3}+6 s t^{4}+50 s t^{3}+122 s t^{2}+78 s t+16 s^{2} t+8 s^{2} t^{2}+8 s^{2}+8 s^{3} \\
{ }^{m} M_{1}(H)= & s\left[\frac{1}{3 s+t+1}+\frac{1}{s+3 t+3}-\frac{1}{4 s+4 t+4}\right]+s t\left[\frac{1}{s+2 t+4}-\frac{1}{4 t+12}+\frac{1}{t+7}+\frac{1}{t+6}+\frac{1}{6}+\frac{1}{7}+\frac{1}{5}\right] \\
& +s t^{2}\left[\frac{1}{4 t+12}\right]+s^{2}\left[\frac{1}{4 s+4 t+4}\right]+s t \frac{1}{3 t+5}+s \frac{1}{2 t+2+2 s}+s t \frac{1}{2 t+6}
\end{aligned}
$$




$$
\begin{aligned}
& R M_{2}(H)=\frac{s^{4}}{2}+\frac{3 s^{3}}{2}+2 s^{2}-s+s^{3} t+9 s^{2} t+22 s t+\frac{39 s t^{2}}{2}+\frac{3 s^{2} t^{2}}{2}+\frac{9 s t^{3}}{2}+\frac{s t^{4}}{2}, \\
& \operatorname{RRR}(H)=s\left[\sqrt{2 s^{2}+2 s t-s-t}+\sqrt{2 s t+2 t^{2}+s+t}-\frac{\sqrt{s^{2}+2 s t+t^{2}}}{2}\right]+s t\left[\sqrt{s t+2 s+t^{2}+2 t}\right. \\
& \left.-\frac{\sqrt{t^{2}+4 t+4}}{2}+\sqrt{3 t+6}+\sqrt{2 t+4}+\sqrt{3}+\sqrt{6}+\sqrt{2}\right]+s t^{2}\left[\frac{\sqrt{t^{2}+4 t+4}}{2}\right] \\
& +s^{2}\left[\frac{\sqrt{s^{2}+2 s t+t^{2}}}{2}\right]+s t \sqrt{(t+2)(2 t+1)}+s \sqrt{(2 t+1)(2 s-1)}+s t \sqrt{(2 t+1)(3)} \text {, } \\
& \operatorname{PGO}(H)=s \frac{1}{\sqrt{\left[6 s^{3}+8 s t^{2}+8 s^{2} t+4 s t+2 s+2 s t^{2}\right]}}+s \frac{1}{\sqrt{\left[2 s^{2} t+8 s t^{2}+10 s t+24 t^{2}+6 t^{3}+18 t+2 s+6\right]}} \\
& +s t \frac{1}{\sqrt{\left[3 s t^{2}+2 t^{3}+12 t^{2}+14 s t+16 t+3 s^{2}+15 s+18\right]}}+\frac{s t^{2}}{2} \frac{1}{\sqrt{\left[2 t^{3}+18 t^{2}+58 t+54\right]}} \\
& +s \frac{1}{\sqrt{[(2 t+2+2 s)(4 s t+4 s)]}}-\frac{s t}{2} \frac{1}{\sqrt{\left[2 t^{3}+18 t^{2}+58 t+54\right]}}+s t \frac{1}{\sqrt{\left[4 t^{2}+40 t+84\right]}} \\
& +s t \frac{1}{\sqrt{[48]}}+s t \frac{1}{\sqrt{[84]}}+\frac{s^{2}}{2} \frac{1}{\sqrt{\left[2 s^{3}+6 s^{2} t+6 s t^{2}+12 s t+8 s^{2}+6 s+4 t^{2}+2 t^{3}+2+6 t\right]}} \\
& -\frac{s}{2} \frac{1}{\sqrt{\left[2 s^{3}+6 s^{2} p+6 s t^{2}+12 s t+8 s^{2}+6 s+4 t^{2}+2 t^{3}+2+6 t\right]}}+s t \frac{1}{\sqrt{\left[3 t^{2}+27 t+54\right]}} \\
& +s t \frac{1}{\sqrt{\left[(3 t+5)\left(2 t^{2}+11 t+11\right)\right]}}+s \frac{1}{\sqrt{[(2 t+2+2 s)(4 s t+4 s)]}}+s t \frac{1}{\sqrt{[(2 t+6)(8 t+8)]}} \\
& +s t \frac{1}{\sqrt{\left[(3 t+5)\left(2 t^{2}+8 t+6\right)\right]}}+s t \frac{1}{\sqrt{[(2 t+6)(8 t+8)]}}+s t \frac{1}{\sqrt{[30]}}, \\
& \mathrm{GO}_{1}(H)=s\left[5 s+2 s^{2}+2 s t+t+1\right]+s\left[3 s+7 t+2 s t+2 t^{2}+5\right]+s t\left[s+2 t+4+s t+t^{2}+t+3 s+3 t+1\right] \\
& \left.+s t \frac{(t-1)}{2}\left[t^{2}+8 t+15\right]\right]+s t[5 t+19]+s t[4 t+15]+s t[14]+s t[21]+s t[11] \\
& +\frac{s(s-1)}{2}\left[4 s+4 t+3+s^{2}+t^{2}+2 s t\right]+s t\left[\left(2 t^{2}+11 t+11\right)\right]+s[(4 s t+2 t+2+6 s)]+s t[(10 t+14)], \\
& \mathrm{GO}_{2}(H)=s^{5}+s^{4}[6]+s^{3}[8]+s[5]+52 s^{2} t+226 s t^{2}+32 s^{2} t^{2}+4 s^{2} t^{3}+29 s^{3} t+92 s t^{3} \\
& +4 s^{3} t^{2}+2 s^{3}+78 s t+8 s^{2} \\
& \mathrm{HGO}_{1}(H)=37 s^{3}+12 s^{2} t+5 s^{2}+24 s^{3} t+s t^{2}+4 s^{2} t^{2}+10 s^{4}+4 s^{5}+6 s^{4} t+2 s^{3} t^{2}+9 s^{3}+62 s^{2} t \\
& +40 s^{2} t^{2}+12 s^{3} t+30 s^{2}+69 s t^{2}+28 s t^{3}+24 s t^{4}+8 s^{2} t^{2}+35 s t+25 s+s t^{5}+2 s^{2} t^{4}+6 s t^{4} \\
& +14 s t^{3}+s^{3} t^{3}+8 s^{3} t^{2}+31 s^{2} t^{2}+16 s^{3} t+63 s t^{2}+42 s^{2} t+49 s t+\frac{s t^{6}}{2}+8 s t^{5}+47 s t^{4}+120 s t^{3} \\
& +\frac{225 s t^{2}}{2}-\frac{s t^{5}}{2}-8 s t^{4}-47 s t^{3}-120 s t^{2}-\frac{225 s t}{2}+25 s t^{3}+190 s t^{2}+361 s t+121 s t+\frac{s^{6}}{2} \\
& +3 s^{4} t^{2}+3 s^{5} t+4 s^{5}+12 s^{4} t+11 s^{4}+27 s^{2} t^{4}+2 s^{2} t^{4}+2 s^{3} t^{3}+12 s^{3} t^{2}+11 s^{2} t^{2}+22 s^{3} t \\
& +2 s^{2} t^{3}+12 s^{2} t+6 s^{3}+\frac{9 t^{2}}{2}-\frac{s^{5}}{2}-3 s^{3} t^{2}-3 s^{4} t-4 s^{4}-12 s^{3} t+11 s^{2} t^{3} \\
& -11 t^{3}-\frac{5 s t^{4}}{2}-2 s^{3} t^{3}-12 s^{2} t^{2}-11 s t^{2}-22 s^{2} t-2 s t^{3}-12 s t-6 s^{2}-\frac{9 t}{2}, \\
& \mathrm{HGO}_{2}(H)=s\left[6 s^{3}+8 s^{2}+8 s^{2} t+4 s t+2 s+2 s t^{2}\right]^{2}+s\left[2 s^{2} t+2 s^{2}+16 s t+8 s t^{2}+18 t^{2}+6 t^{3}\right. \\
& +18 t+8 s+6]^{2}+s t\left[3 s t^{2}+2 t^{3}+12 t^{2}+14 s t+s^{2} t+15 s+12+12 t\right]^{2}+\frac{s t^{2}}{2}\left[2 t^{3}\right.
\end{aligned}
$$




$$
\begin{aligned}
& \left.+18 t^{2}+54 t+54\right]^{2}-\frac{s t}{2}\left[2 t^{3}+18 t^{2}+54 t+54\right]^{2}+s t\left[4 t^{2}+40 t+84\right]^{2}+s t\left[3 t^{2}+27 t+54\right]^{2} \\
& +s t[48]^{2}+s t[84]^{2}+s t[30]^{2}+\frac{s^{2}}{2}\left[2 s^{3}+6 s^{2} t+6 s t^{2}+12 s t+6 s^{2}+6 s+6 t^{2}+2 t^{3}+2+6 t\right]^{2} \\
& -\frac{s}{2}\left[2 t^{3}+6 s^{2} t+6 s t^{2}+12 s t+6 s^{2}+6 s+6 t^{2}+2 t^{3}+2+6 t\right]^{2},
\end{aligned}
$$

$$
\begin{aligned}
& \operatorname{SGO}(H)=s \frac{1}{\sqrt{\left[3 s+t+1+2 s^{2}+2 s t+2 s\right]}}+s \frac{1}{\sqrt{\left[s+3 t+3+2 s+4 t+2 t^{2}+2\right]}} \\
& +\frac{s t^{2}}{2} \frac{1}{\sqrt{\left[t^{2}+6 t+9+2 t+6\right]}}-\frac{s t}{2 \sqrt{t^{2}+6 t+9+2 t+6}}+s t \frac{1}{\sqrt{[5 t+12+7]}} \\
& +s t \frac{1}{\sqrt{[4 t+15]}}+s t \frac{1}{\sqrt{[14]}}+s t \frac{1}{\sqrt{[19]}}+s t \frac{1}{\sqrt{[11]}}+s t \frac{1}{\sqrt{[(10 t+14)]}} \\
& +\frac{s^{2}}{2} \frac{1}{\sqrt{\left[s^{2}+2 s t+2 s+2 t+t^{2}+1+2 s+2 t+2\right]}}-\frac{s}{2} \frac{1}{\sqrt{\left[s^{2}+2 s t+2 s+2 t+t^{2}+1+2 s+2 t+2\right]}} \\
& +s t \frac{1}{\sqrt{\left[\left(2 t^{2}+11 t+11\right)\right]}}+s \frac{1}{\sqrt{[(4 s t+2 t+2+6 s)]}}+s t \frac{1}{\sqrt{\left[t^{2}+s t+4 t+3 s+3+s+4\right]}}, \\
& F(H)=4 s^{3}+s^{3}+s t^{2}+s+2 s t+2 s^{2} t+2 s^{2}+s^{3}+s t^{2}+s+2 s t+2 s^{2} t+2 s^{2}+4 s t^{2}+4 s+8 s t \\
& +s^{3} t+s t^{3}+s t+2 s t^{2}+2 s^{2} t+2 s^{2} t^{2}+s t^{3}+9 s t+6 s t^{2}+\frac{s t^{4}}{2}+\frac{9 s t^{2}}{2}+3 s t^{3}+\frac{s t^{4}}{2} \\
& +\frac{9 s t^{2}}{2}+3 s t^{3}-\frac{s t^{3}}{2}-3 s t^{2}-\frac{9 s t}{2}-3 s t^{2}-\frac{s t^{3}}{2}-\frac{9 s t}{2}+s t^{3}+9 s t+6 s t^{2}+16 s t+s t^{3} \\
& +9 s t+6 s t^{2}+9 s t+20 s t+28 s t+13 s t+\frac{s^{4}}{2}+s \frac{{ }^{2} t^{2}}{2}+\frac{s^{2}}{2}+s^{2} t+s^{3}+s^{3} t-\frac{s^{3}}{2}-\frac{s t^{2}}{2} \\
& -\frac{s}{2}-s t-s^{2}-\frac{s^{3}}{2}-\frac{s t^{2}}{2}-2 s t-\frac{s t}{2}-s^{2}-s^{2} t+s t\left[5 t^{2}+14 t+13\right]+s\left[4 t^{2}+4+8 t+4 s^{2}\right] \\
& +s t\left[4 t^{2}+20+8 t\right] \text {. }
\end{aligned}
$$

Proof. For line classification shown in Table 1, use of formulas (5)-(9), (11)-(18), and (22) will give desired above expressions.
Theorem 3. Let $H$ be the total graph of a Kragujevac tree; $K \in K g_{q=s(2 t+1)+1, s}$, then M-polynomial and certain topological indices deducted from M-polynomial are

$$
\begin{aligned}
M(H ; u, v)= & s u^{2 s} v^{s+t+1}+s u^{s+t+1} v^{2 t+2}+s t u^{s+t+1} v^{t+3}+\frac{s t^{2}}{2} u^{t+3} v^{t+3}-\frac{s t}{2} u^{t+3} v^{t+3} \\
& +s t u^{t+3} v^{4}+s t u^{t+3} v^{3}+s t u^{4} v^{2}+s t u^{4} v^{3}+s t u^{3} v^{2}+\frac{s^{2}}{2} u^{s+t+1} v^{s+t+1} \\
& -\frac{s}{2} u^{s+t+1} v^{s+t+1}+s t u^{t+3} v^{2 t+2}+s u^{2 t+2} v^{2 s}+s t u^{2 t+2} v^{4} \\
M_{1}(H)= & 6 s^{2}+\frac{95}{2} s t+5 s+2 s^{2} t+11 s t^{2}+s t^{3}+s^{3}, \\
M_{2}(H)= & \frac{5 s^{3}}{2}+7 s^{2} t+\frac{7 s^{2}}{2}+14 s t^{2}+\frac{97 s t}{2}+\frac{3}{2} s+\frac{3 s^{2} t^{2}}{2}+\frac{7 s t^{3}}{2}
\end{aligned}
$$




$$
\begin{aligned}
& +\frac{s t^{4}}{2}+\frac{s^{4}}{2}+s^{3}+16 s t^{2}+14 s t+4 s^{2}+2 s t^{3}+4 s^{2} t \\
m_{2} M_{2}(H)= & \frac{1}{2 s+2 t+2}+s\left[\frac{1}{2 t^{2}+2 s t+4 t+2 s+2}-\frac{1}{2(s+t+1)^{2}}\right]+s t\left[\frac{1}{t^{2}+s t+4 t+3 s+3}\right. \\
& \left.-\frac{1}{2 t^{2}+2 t+18}+\frac{1}{4 t+12}+s t \frac{1}{(2 t+2)(4)}+\frac{s t}{8}+\frac{1}{3 t+9}+\frac{1}{8}+\frac{1}{12}+\frac{1}{6}\right] \\
& +s t^{2}\left[\frac{1}{2 t^{2}+12 t+18}\right]+s^{2}\left[\frac{1}{2(s+t+1)^{2}}\right]+s t \frac{1}{(2 t+2)(t+3)}+s \frac{1}{(2 t+2)(2 s)^{2}} \\
R_{\alpha}(H)= & \frac{s}{(2 s)^{\alpha}(s+t+1)^{\alpha}}+\frac{s}{(2 t+2)^{\alpha}(s+t+1)^{\alpha}}+\frac{s t}{(t+3)^{\alpha}(s+t+1)^{\alpha}}+\frac{(s t)^{2}}{2(t+3)^{\alpha}(t+3)^{\alpha}} \\
& -\frac{s t}{2(t+3)^{\alpha}(t+3)^{\alpha}}+\frac{s t}{(4)^{\alpha}(t+3)^{\alpha}}+\frac{s t}{(3)^{\alpha}(t+3)^{\alpha}}+\frac{s t}{(3)^{\alpha}(4)^{\alpha}}+\frac{s t}{(2)^{\alpha}(3)^{\alpha}} \\
& +\frac{s t}{(4)^{\alpha}(2)^{\alpha}+\frac{s}{2(s+t+1)^{\alpha}(s+t+1)^{\alpha}}-\frac{1}{2(s+t+1)^{\alpha}(s+t+1)^{\alpha}}+s t \frac{1}{(2 t+2)^{\alpha}(t+3)^{\alpha}}} \\
& +s \frac{1}{(2 s)^{\alpha}(2 t+2)^{\alpha}}+s t \frac{1}{(4)^{\alpha}(2 t+2)^{\alpha}},
\end{aligned}
$$

$$
\begin{aligned}
& \mathrm{RR}_{\alpha}(H)=\left[s(2 s)^{\alpha}(s+t+1)^{\alpha}+s(s+t+1)^{\alpha}(2 t+2)^{\alpha}+s t(s+t+1)^{\alpha}(t+3)^{\alpha}\right. \\
& -\frac{s}{2}(s+t+1)^{\alpha}(s+t+1)^{\alpha}+\frac{s t^{2}}{2}(t+3)^{\alpha}(t+3)^{\alpha}-\frac{s t}{2}(t+3)^{\alpha}(t+3)^{\alpha}+s t(t+3)^{\alpha}(4)^{\alpha} \\
& +s t(t+3)^{\alpha}(3)^{\alpha}+s t(4)^{\alpha}(2)^{\alpha}+s t(4)^{\alpha}(3)^{\alpha}+s t(3)^{\alpha}(2)^{\alpha}+\frac{s^{2}}{2}(s+t+1)^{\alpha}(s+t+1)^{\alpha} \\
& +s t(2 t+2)^{\alpha}(t+3)^{\alpha}+s(2 s)^{\alpha}(2 t+2)^{\alpha}+s t(4)^{\alpha}(2 t+2)^{\alpha} \text {, } \\
& \operatorname{SDD}(H)=s^{2}\left[\frac{2}{s+t+1}+\frac{1}{2 t+2}+1\right]+s t\left[\frac{1}{2 t+2}+\frac{2}{t+3}+\frac{2}{s+t+1}+\frac{3}{2(t+3)}+\frac{29}{4}\right] \\
& +s^{2} t\left[\frac{1}{t+3}\right]+\frac{1}{2 t+2}+s t^{2}\left[\frac{1}{t+3}+\frac{1}{s+t+1}+\frac{1}{t+3}+\frac{13}{12}\right]+s t^{3}\left[\frac{1}{2(t+3)}\right] \\
& +\frac{1}{2}+\frac{t}{2}+s\left[\frac{1}{s+t+1}+\frac{1}{2}\right]+2 s t \\
& H(H)=s\left[\frac{2}{3 s+t+1}+\frac{2}{s+3 t+4}-\frac{1}{2 s+2 t+2}\right]+s t\left[\frac{2}{s+2 t+4}-\frac{1}{2 t+6}+\frac{2}{t+7}+\frac{2}{t+6}\right. \\
& \left.+\frac{1}{3}+\frac{2}{7}+\frac{2}{5}\right]+s^{2}\left[\frac{1}{2 s+2 t+2}\right]+s t^{2}\left[\frac{1}{2 t+6}\right]+s t \frac{2}{3 t+5}+s \frac{2}{2 t+2+2 s}+s t \frac{2}{2 t+6}, \\
& \operatorname{ISI}(H)=s(2 s)(s+t+1) \frac{1}{3 s+t+1}+s(s+t+1)(2 t+2) \frac{1}{s+3 t+3}+s t(s+t+1)(t+3) \frac{1}{s+2 t+4} \\
& +\frac{s t^{2}}{2}(t+3)(t+3) \frac{1}{2 t+6}+s t(4)(3) \frac{1}{7}+s t(3)(2) \frac{1}{5}-\frac{s t}{2}(t+3)(t+3) \frac{1}{2 t+6} \\
& +s t(t+3)(4) \frac{1}{t+7}+s t(t+3)(3) \frac{1}{t+6}+s t(4)(2) \frac{1}{6}+\frac{s^{2}}{2}(s+t+1)(s+t+1) \frac{1}{2 s+2 t+2} \\
& -\frac{s}{2}(s+t+1)(s+t+1) \frac{1}{2 s+2 t+2}+s t(t+3) \frac{2 t+2}{3 t+5}+s(2 t+2) \frac{2 s}{2 t+2 s+2} \\
& +s t(2 t+2) \frac{4}{2 t+6} \\
& A(H)=s(2 s)^{3}(s+t+1)^{3} \frac{1}{(3 s+t-1)^{3}}+s(s+t+1)^{3}(2 t+2)^{3} \frac{1}{(s+3 t+1)^{3}}
\end{aligned}
$$




$$
\begin{aligned}
& +s t(s+t+1)^{3}(t+3)^{3} \frac{1}{(s+2 t+2)^{3}}+\frac{s t^{2}}{2}(t+3)^{3}(t+3)^{3} \frac{1}{(2 t+4)^{3}}-\frac{s t}{2}(t+3)^{3}(t+3)^{3} \frac{1}{(2 t+4)^{3}} \\
& +s t(t+3)^{3}(4)^{3} \frac{1}{(t+5)^{3}}+s t(t+3)^{3}(3)^{3} \frac{1}{(t+4)^{3}}+s t(4)^{3}(2)^{3} \frac{1}{(4)^{3}}+s t(4)^{3}(3)^{3} \frac{1}{(5)^{3}} \\
& +s t(3)^{3}(2)^{3} \frac{1}{(3)^{3}}+\frac{s^{2}}{2}(s+t+1)^{3}(s+t+1)^{3} \frac{1}{(2 s+2 t)^{3}}-\frac{s}{2}(s+t+1)^{3}(s+t+1)^{3} \frac{1}{(2 s+2 t)^{3}} \\
& +s t(2 t+2)^{3}(2 t+3)^{3} \frac{1}{(3 t+3)^{3}}+s(2 s)^{3}(2 t+2)^{3} \frac{1}{(2 t+2 s)^{3}}+s t(4)^{3}(2 t+2)^{3} \frac{1}{(2 t+4)^{3} .}
\end{aligned}
$$

Proof. For line distribution shown in Table 1, use of formulas (19)-(28) will give above desired expressions.

\section{Certain Topological Indices and \\ Polynomials of $L(T(K))$, \\ where $K \in K g_{q==s(2 t+1)+1, s}$}

In this section, we calculate specific topological indices and polynomials and many other chemical indices in terms of these polynomials, as presented in the first section of the Kragujevac tree total line graph. Figures 4-7 represents certain topological indices and polynomials.

Theorem 4. Let $H$ be the line graph of total of Kragujevac tree; $K \in K g_{q=s(2 t+1)+1, s}$. Then,

$$
\begin{aligned}
& F(H, u)=\sum_{m l \in E(H)} u^{\left(d_{m}\right)^{2}+\left(d_{l}\right)^{2}} \\
& +s t u^{(t+5)^{2}+(3 t+3)^{2}}+s t(s-1) u^{(t+5)^{2}+(2 t+4)^{2}}+s t(s-1) u^{(t+4)^{2}+(2 t+4)^{2}}+s t u^{(4)^{2}+(5)^{2}} \\
& +s t u^{(t+4)^{2}+(3 t+3)^{2}}+s t u^{(t+4)^{2}+(2 t+s+2)^{2}}+s t u^{(t+4)^{2}+(5)^{2}}+s t u^{(t+4)^{2}+(3)^{2}} \\
& +s t u^{(t+5)^{2}+(5)^{2}}+s t u^{(5)^{2}+(3)^{2}}+s t u^{(2 t+s+3)^{2}+(3 t+3)^{2}}+s t(t-1) u^{(3 t+3)^{2}+(2 t+4)^{2}} \\
& +s t(t-1) u^{(2 t+s+2)^{2}+(2 t+4)^{2}}+\frac{s t(t-1)(t-2)}{2} u^{(2 t+4)^{2}+(2 t+4)^{2}}+s t u^{(4)^{2}+(3)^{2}} \\
& +s t u^{(4)^{2}+(t+5)^{2}}+s t u^{(2 t+4)^{2}+(4)^{2}}+s t u^{(2 t+4)^{2}+(5)^{2}}+s t u^{(2 t+s+2)^{2}+(s+3 t+1)^{2}}+\frac{s t(t-1)}{2} \\
& \cdot u^{(2 t+s+2)^{2}+(2 t+s+2)^{2}}+s t u^{(3 s+t-1)^{2}+(2 t+s+2)^{2}}+s u^{(3 s+t-1)^{2}+(s+3 t+1)^{2}}+s t(s-1) \\
& \cdot u^{(2 t+s+2)^{2}+(2 s+2 t)^{2}}+\frac{s(s-1)(s-2)}{2} u^{(2 s+2 t)^{2}+(2 s+2 t)^{2}}+s(s-1) u^{(3 s+t-1)^{2}+(2 s+2 t)^{2}} \\
& +s(s-1) u^{(s+3 t+1)^{2}+(2 s+2 t)^{2}}+s(s-1) u^{(2 s+2 t)^{2}+(2 s+2 t)^{2}} \\
& +\frac{s(s-1)}{2} u^{(3 s+t-1)^{2}+(3 s+t-1)^{2}}+s(s-1) u^{(2 s+2 t)^{2}+(3 s+t-1)^{2}} \\
& +(s-1) u^{(2 s+2 t)^{2}+(2 s+2 t)^{2}}+s t u^{(2 t+4)^{2}+(s+3 t+1)^{2}}+s t u^{(2 t+4)^{2}+(2 s+2 t)^{2}} \\
& +\frac{s t(t-1)}{2} u^{(3 t+3)^{2}+(3 t+3)^{2}}+s t u^{(3 t+3)^{2}+(s+3 t+1)^{2}} \\
& +s t u^{(3 t+3)^{2}+(2 s+2 t)^{2}}+s u^{(s+3 t+1)^{2}+(2 s+2 t)^{2}},
\end{aligned}
$$




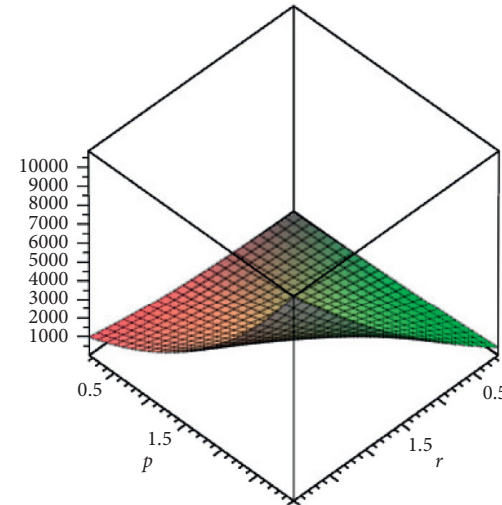

HM $(\mathrm{H})$

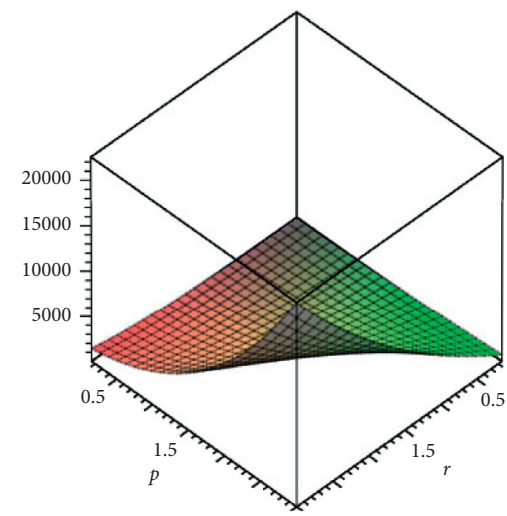

$\operatorname{ReM}_{3}(\mathrm{H})$

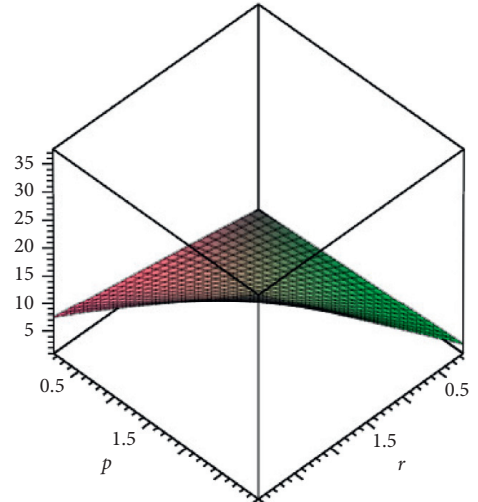

$\operatorname{ReM}_{1}(\mathrm{H})$

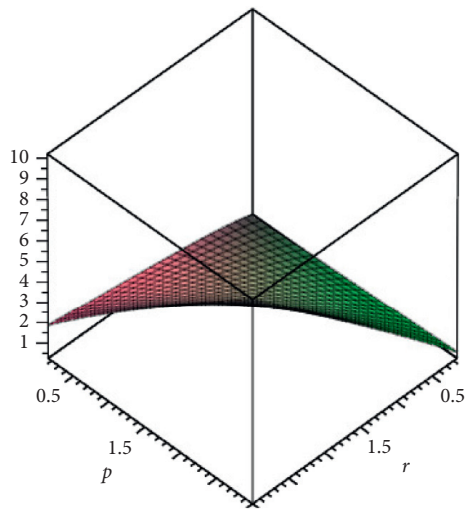

$\mathrm{m}_{\mathrm{M} 1}(\mathrm{H})$

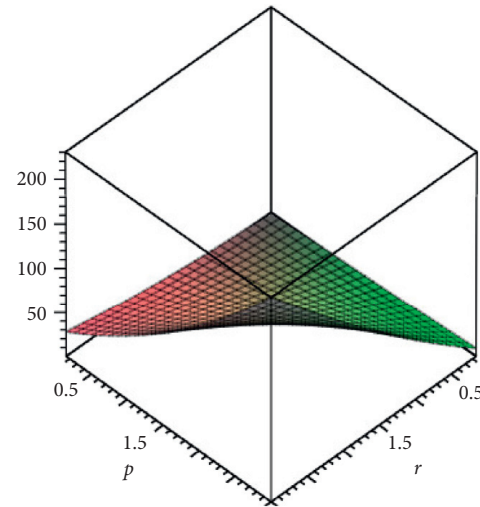

$\operatorname{ReM}_{2}(\mathrm{H})$

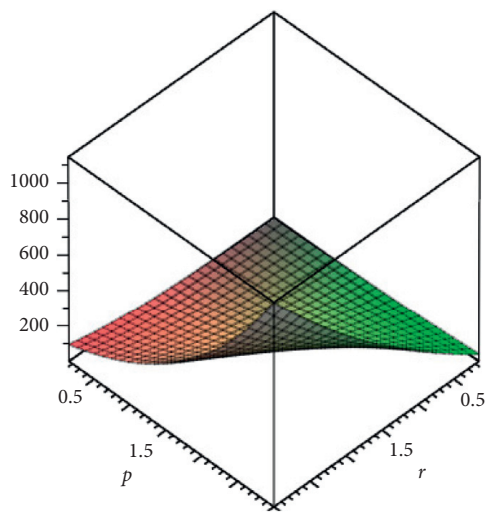

$\mathrm{RM}_{2}(\mathrm{H})$

Figure 4: The pictorial representation of (a) $H M(H)$, (b) $\operatorname{Re} M_{1}(H),(\mathrm{c}) \operatorname{Re} M_{2}(H)$, (d) $\operatorname{Re} M_{3}(H)$, (e) ${ }^{m} M_{1}(H)$, and (f) $R M_{2}(H)$ in space.

$$
\begin{aligned}
M_{1}(H, u)= & \sum_{m l \in E(H)} u^{d_{m}+d_{l}} \\
& +s t u^{(t+5)+(3 t+3)}+s t(s-1) u^{(t+5)+(2 t+4)}+s t(s-1) u^{(t+4)+(2 t+4)}+s t u^{(t+4)+(3 t+3)} \\
& +s t u^{(t+4)+(2 t+s+2)}+s t u^{(t+4)+(5)}+s t u^{(t+4)+(3)}+s t u^{(t+5)+(5)}+s t u^{(5)+(3)} \\
& +s t u^{(2 t+s+3)+(3 t+3)}+s t(t-1) u^{(3 t+3)+(2 t+4)}+s t(t-1) u^{(2 t+s+2)+(2 t+4)} \\
& +s u^{(s+3 t+1)+(2 s+2 t)}+\frac{s t(t-1)(t-2)}{2} u^{(2 t+4)+(2 t+4)}+s t u^{(4)+(3)}+s t u^{(4)+(5)} \\
& +s t u^{(4)+(t+5)}+s t u^{(2 t+4)+(4)}+s t u^{(2 t+4)+(5)}+s t u^{(2 t+s+2)+(s+3 t+1)}+\frac{s t(t-1)}{2} \\
& \cdot u^{(2 t+s+2)+(2 t+s+2)}+s t u^{(3 s+t-1)+(2 t+s+2)}+s u^{(3 s+t-1)+(s+3 t+1)}+s t(s-1) \\
& \cdot u^{(2 t+s+2)+(2 s+2 t)}+\frac{s(s-1)(s-2)}{2} u^{(2 s+2 t)+(2 s+2 t)}+s(s-1) u^{(3 s+t-1)+(2 s+2 t)} \\
& +s(s-1) u^{(s+3 t+1)+(2 s+2 t)}+s(s-1) u^{(2 s+2 t)+(2 s+2 t)}+\frac{s(s-1)}{2} u^{(3 s+t-1)+(3 s+t-1)} \\
& +s(s-1) u^{(2 s+2 t)+(3 s+t-1)}+(s-1) u^{(2 s+2 t)+(2 s+2 t)}+s t u^{(2 t+4)+(s+3 t+1)}+s t \\
& \cdot u^{(2 t+4)+(2 s+2 t)}+\frac{s t(t-1)}{2} u^{(3 t+3)+(3 t+3)}+s t u^{(3 t+3)+(s+3 t+1)}+s t u^{(3 t+3)+(2 s+2 t)}
\end{aligned}
$$




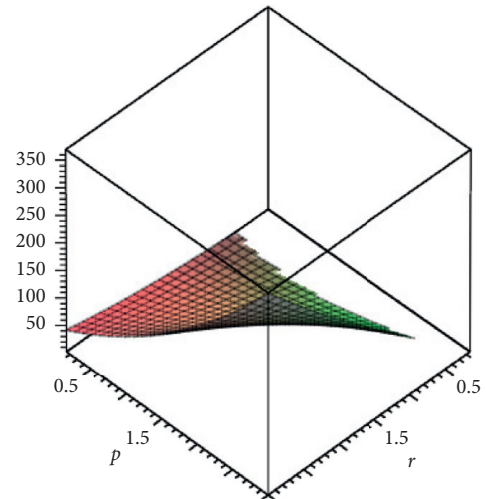

RRR (H)

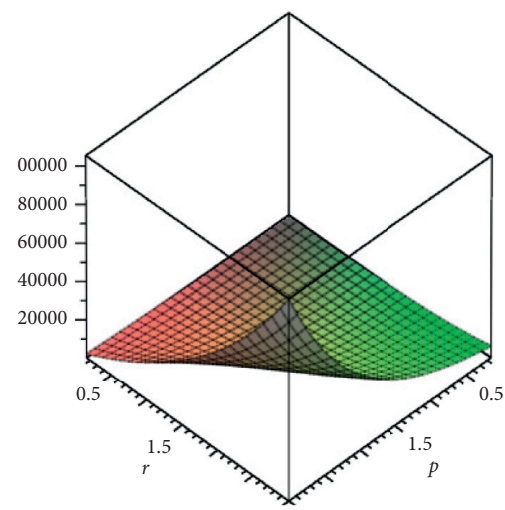

$\mathrm{HGO}_{1}(\mathrm{H})$

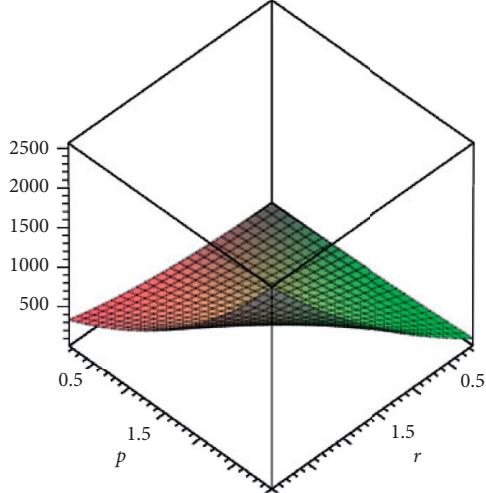

$\mathrm{GO}_{1}(\mathrm{H})$

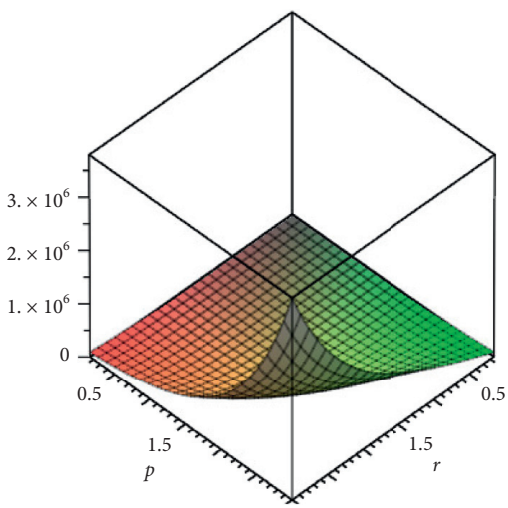

$\mathrm{HGO}_{2}(\mathrm{H})$

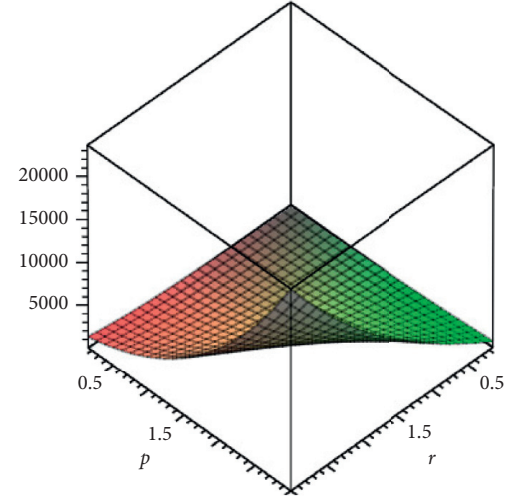

$\mathrm{GO}_{2}(\mathrm{H})$

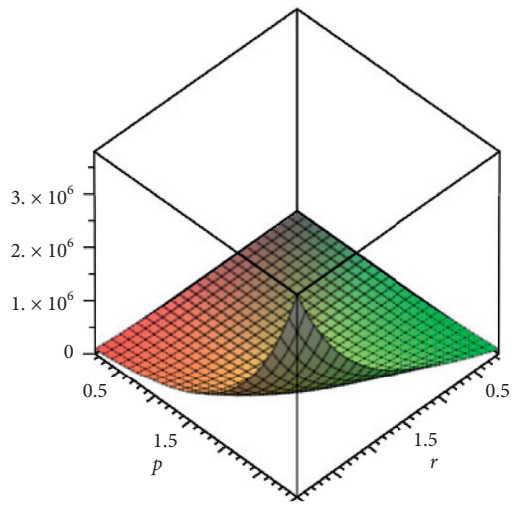

PGO (H)

Figure 5: The pictorial characterization of (a) $\mathrm{RRR}(H)$, (b) $\mathrm{GO}_{1}(H)$, (c) $\mathrm{GO}_{2}(H)$, (d) $\mathrm{HGO}_{1}(H)$, (e) $\mathrm{HGO}_{2}(H)$, and (f) PGO (H) in space.

$$
\begin{aligned}
M_{2}(H, u)= & \sum_{m l \in E(H)} u^{d_{m} d_{l}} \\
& +s t u^{(t+5) \times(3 t+3)}+s t(s-1) u^{(t+5) \times(2 t+4)}+s t(s-1) u^{(t+4) \times(2 t+4)} \\
& +s t u^{(t+4) \times(3 t+3)}+s t u^{(t+4) \times(2 t+s+2)}+s t u^{(t+4) \times(5)}+s t u^{(t+4) \times(3)} \\
& +s t u^{(t+5) \times(5)}+s t u^{(5) \times(3)}+s t u^{(2 t+s+3) \times(3 t+3)}+s t(t-1) u^{(3 t+3) \times(2 t+4)} \\
& +s t(t-1) u^{(2 t+s+2) \times(2 t+4)}+\frac{s t(t-1)(t-2)}{2} u^{(2 t+4) \times(2 t+4)}+s t u^{(4) \times(3)} \\
& +s t u^{(4) \times(5)}+s t u^{(4) \times(t+5)}+s t u^{(2 t+4) \times(4)}+s t u^{(2 t+4) \times(5)}+s t u^{(2 t+s+2) \times(s+3 t+1)} \\
& +\frac{s t(t-1)}{2} u^{(2 t+s+2) \times(2 t+s+2)}+s t u^{(3 s+t-1) \times(2 t+s+2)}+s u^{(3 s+t-1) \times(s+3 t+1)} \\
& +s t(s-1) u^{(2 t+s+2) \times(2 s+2 t)}+\frac{s(s-1)(s-2)}{2} u^{(2 s+2 t) \times(2 s+2 t)}+s(s-1) \\
& \cdot u^{(3 s+t-1) \times(2 s+2 t)}+s(s-1) u^{(s+3 t+1) \times(2 s+2 t)}+s(s-1) u^{(2 s+2 t) \times(2 s+2 t)}+\frac{s(s-1)}{2} \\
& \cdot u^{(3 s+t-1) \times(3 s+t-1)}+s(s-1) u^{(2 s+2 t) \times(3 s+t-1)}+(s-1) u^{(2 s+2 t) \times(2 s+2 t)} \\
& +s t u^{(2 t+4) \times(s+3 t+1)}+s t u^{(2 t+4) \times(2 s+2 t)}+\frac{s t(t-1)}{2} u^{(3 t+3) \times(3 t+3)} \\
& +s t u^{(3 t+3) \times(s+3 t+1)}+s t u^{(3 t+3) \times(2 s+2 t)}+s u^{(s+3 t+1) \times(2 s+2 t)},
\end{aligned}
$$




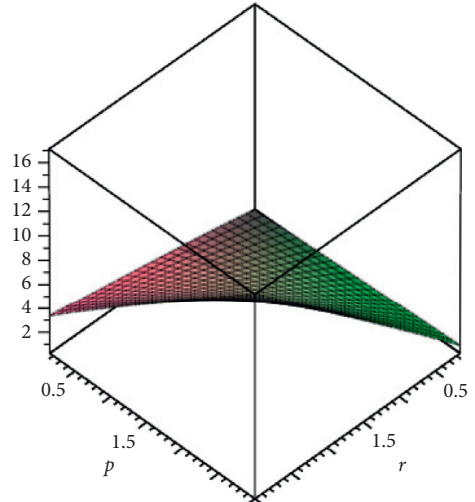

SGO $(\mathrm{H})$

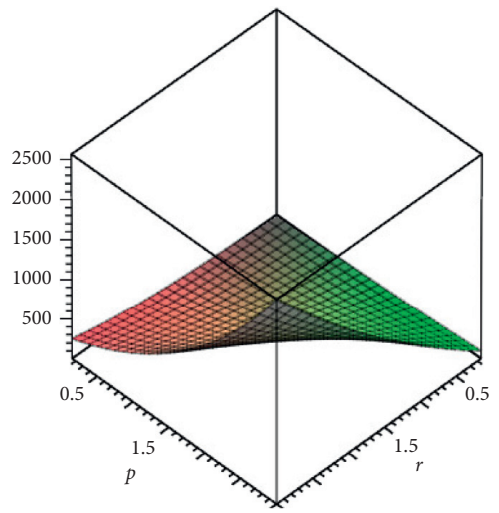

$\mathrm{M}_{2}(\mathrm{H})$

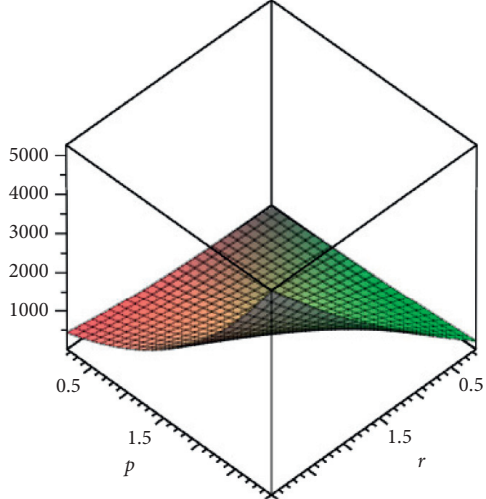

$\mathrm{F}(\mathrm{H})$

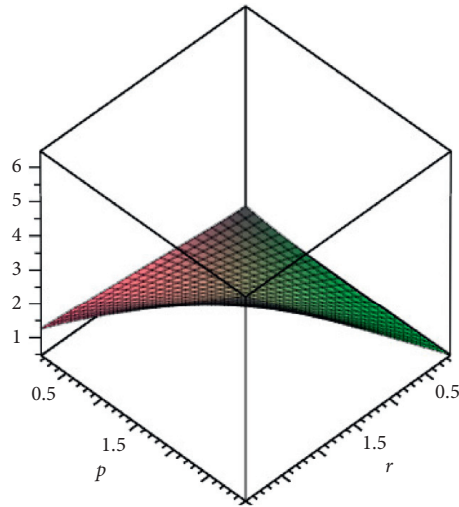

$\mathrm{m}_{\mathrm{M}_{2}(\mathrm{H})}$

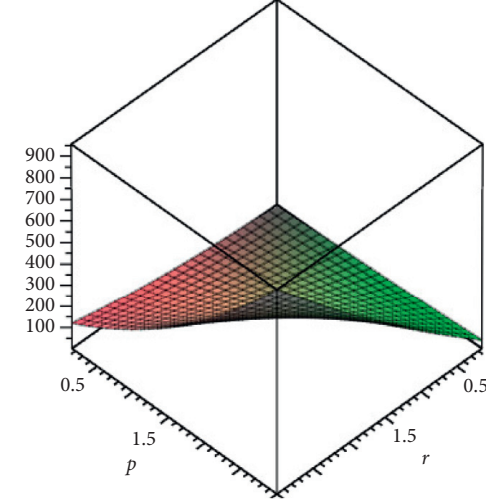

$\mathrm{M}_{1}(\mathrm{H})$

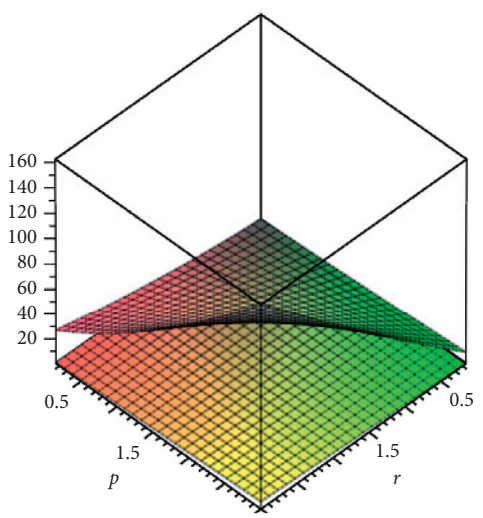

$\operatorname{SDD}(\mathrm{H})$

Figure 6: The pictorial representation of (a) $\mathrm{SGO}(H)$, (b) $F(H)$, (c) $M_{1}(H),\left(\right.$ d) $M_{2}(H)$, (e) ${ }^{m} M_{2}$, and (f) $\mathrm{SDD}(H)$ in space.

$$
\begin{aligned}
& M_{3}(H, u)=\sum_{m l \in E(H)} u^{\left|d_{m}-d_{l}\right|} \\
& +s t u^{(t+5)-(3 t+3)}+s t(s-1) u^{(t+5)-(2 t+4)}+s t(s-1) u^{(t+4)-(2 t+4)}+s t u^{(t+4)-(3 t+3)} \\
& +s t u^{(t+4)-(2 t+s+2)}+s t u^{(t+4)-(5)}+s t u^{(t+4)-(3)}+s t u^{(t+5)-(5)}+s t u^{(5)-(3)} \\
& +s t u^{(2 t+s+3)-(3 t+3)}+s t(t-1) u^{(3 t+3)-(2 t+4)}+s t(t-1) u^{(2 t+s+2)-(2 t+4)}+\frac{s t(t-1)(t-2)}{2} \\
& \cdot u^{(2 t+4)-(2 t+4)}+s t u^{(4)-(3)}+s t u^{(4)-(5)}+s t u^{(4)-(t+5)}+s t u^{(2 t+4)-(4)}+s t u^{(2 t+4)-(5)} \\
& +s t u^{(2 t+s+2)-(s+3 t+1)}+\frac{s t(t-1)}{2} u^{(2 t+s+2)-(2 t+s+2)}+s t u^{(3 s+t-1)-(2 t+s+2)} \\
& +s u^{(3 s+t-1)-(s+3 t+1)}+s t(s-1) u^{(2 t+s+2)-(2 s+2 t)}+\frac{s(s-1)(s-2)}{2} u^{(2 s+2 t)-(2 s+2 t)} \\
& +s(s-1) u^{(3 s+t-1)-(2 s+2 t)}+s(s-1) u^{(s+3 t+1)-(2 s+2 t)}+s(s-1) u^{(2 s+2 t)-(2 s+2 t)} \\
& +\frac{s(s-1)}{2} u^{(3 s+t-1)-(3 s+t-1)}+s(s-1) u^{(2 s+2 t)-(3 s+t-1)}+(s-1) u^{(2 s+2 t)-(2 s+2 t)} \\
& +s t u^{(2 s+2 t)-(s+3 t+1)}+s t u^{(2 t+4)-(2 s+2 t)}+\frac{s t(t-1)}{2} u^{(3 t+3)-(3 t+3)}+s t u^{(3 t+3)-(s+3 t+1)} \\
& +s t u^{(3 t+3)-(2 s+2 t)}+s u^{(s+3 t+1)-(2 s+2 t)} .
\end{aligned}
$$




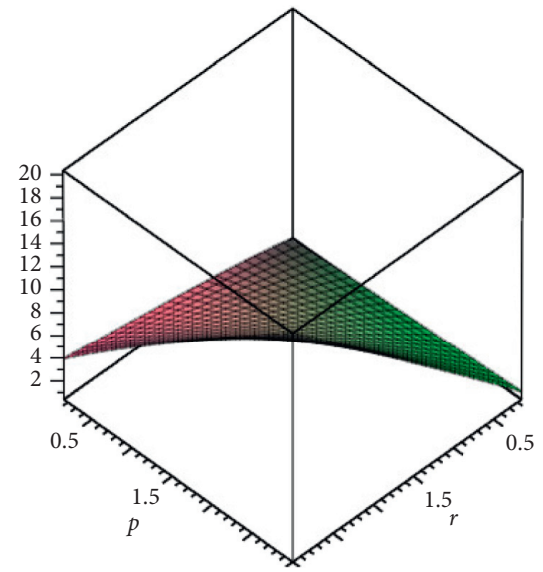

$\mathrm{H}(\mathrm{H})$

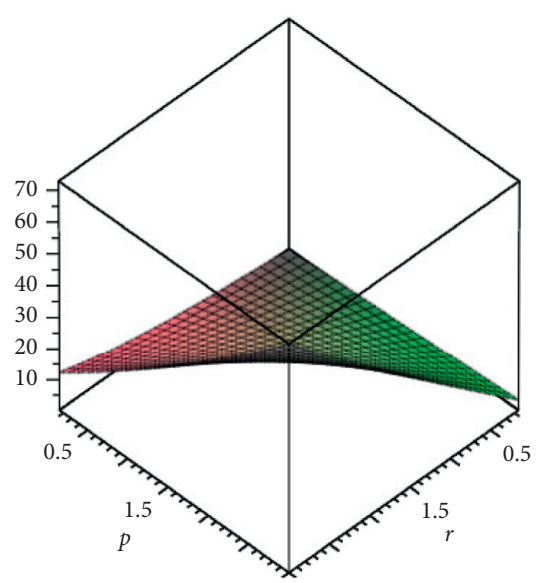

ISI (H)

FIgURE 7: The pictorial characterization of (a) $H(H)$ and (b) ISI $(H)$ in space.

Proof. Consider the line graph of total graph of Kragujevac tree, denoted by $L(T(K))$. We categorize the lines of
$L(T(K))$ based on the lines of type $E_{\left(d_{m}, d_{l}\right)}$, where $m l \in E(L(T(K)))$. The line distribution is shown in Table 2 .

$$
\begin{aligned}
& F(H, u)=\sum_{m l \in E(H)} u^{\left(d_{m}\right)^{2}+\left(d_{l}\right)^{2}} \\
& +s t u^{(t+5)^{2}+(3 t+3)^{2}}+s t(s-1) u^{(t+5)^{2}+(2 t+4)^{2}}+s t(s-1) u^{(t+4)^{2}+(2 t+4)^{2}}+s t u^{(4)^{2}+(5)^{2}} \\
& +s t u^{(2 t+4)^{2}+(3 t+3)^{2}}+s t u^{(t+4)^{2}+(2 t+s+2)^{2}}+s t u^{(t+4)^{2}+(5)^{2}}+s t u^{(t+4)^{2}+(3)^{2}} \\
& +s t u^{(t+5)^{2}+(5)^{2}}+s t u^{(5)^{2}+(3)^{2}}+s t u^{(2 t+s+3)^{2}+(3 t+3)^{2}}+s t(t-1) u^{(3 t+3)^{2}+(2 t+4)^{2}} \\
& +s t(t-1) u^{(2 t+s+2)^{2}+(2 t+4)^{2}}+\frac{s t(t-1)(t-2)}{2} u^{(2 t+4)^{2}+(2 t+4)^{2}}+s t u^{(4)^{2}+(3)^{2}} \\
& +s t u^{(4)^{2}+(t+5)^{2}}+s t u^{(2 t+4)^{2}+(4)^{2}}+s t u^{(2 t+4)^{2}+(5)^{2}}+s t u^{(2 t+s+2)^{2}+(s+3 t+1)^{2}} \\
& +\frac{s t(t-1)}{2} u^{(2 t+s+2)^{2}+(2 t+s+2)^{2}}+s t u^{(3 s+t-1)^{2}+(2 t+s+2)^{2}}+s u^{(3 s+t-1)^{2}+(s+3 t+1)^{2}} \\
& +s t(s-1) u^{(2 t+s+2)^{2}+(2 s+2 t)^{2}}+\frac{s(s-1)(s-2)}{2} u^{(2 s+2 t)^{2}+(2 s+2 t)^{2}}+s(s-1) u^{(3 s+t-1)^{2}+(2 s+2 t)^{2}} \\
& +s(s-1) u^{(s+3 t+1)^{2}+(2 s+2 t)^{2}}+s(s-1) u^{(2 s+2 t)^{2}+(2 s+2 t)^{2}} \\
& +\frac{s(s-1)}{2} u^{(3 s+t-1)^{2}+(3 s+t-1)^{2}}+s(s-1) u^{(2 s+2 t)^{2}+(3 s+t-1)^{2}} \\
& +(s-1) u^{(2 s+2 t)^{2}+(2 s+2 t)^{2}}+s t u^{(2 t+4)^{2}+(s+3 t+1)^{2}}+s t u^{(2 t+4)^{2}+(2 s+2 t)^{2}} \\
& +\frac{s t(t-1)}{2} u^{(3 t+3)^{2}+(3 t+3)^{2}}+s t u^{(3 t+3)^{2}+(s+3 t+1)^{2}} \\
& +s t u^{(3 t+3)^{2}+(2 s+2 t)^{2}}+s u^{(s+3 t+1)^{2}+(2 s+2 t)^{2}},
\end{aligned}
$$


TABLE 2: Line classification of $L(T(K))$.

\begin{tabular}{|c|c|}
\hline Lines of type & Number of lines \\
\hline$E_{(t+5, t+4)}$ & st \\
\hline$E_{(2 t+4, t+5)}$ & $s t$ \\
\hline$E_{(t+5,2 t+s+2)}$ & $s t$ \\
\hline$E_{(t+5,3 t+3)}$ & $s t$ \\
\hline$E_{(t+5,2 t+4)}$ & $s t(s-1)$ \\
\hline$E_{(t+4,2 t+4)}$ & $s t(s-1)$ \\
\hline$E_{(t+4,3 t+3)}$ & st \\
\hline$E_{(t+4,2 t+s+2)}$ & $s t$ \\
\hline$E_{(t+4,5)}$ & $s t$ \\
\hline$E_{(t+4,3)}$ & st \\
\hline$E_{(t+5,5)}$ & st \\
\hline$E_{(5,3)}$ & st \\
\hline$E_{(2 t+s+2,3 t+3)}$ & st \\
\hline$E_{(3 t+3,2 t+4)}$ & $s t(s-1)$ \\
\hline$E_{(2 t+s+2,2 t+4)}^{(t i+3, t+4)}$ & $s t(s-1)$ \\
\hline$E_{(2 t+4,2 t+4)}$ & $(s t(t-1)(t-2)) / 2$ \\
\hline$E_{(4,3)}$ & $s t$ \\
\hline$E_{(4,5)}$ & $s t$ \\
\hline$E_{(4, t+5)}^{(4,5)}$ & st \\
\hline$E_{(2 t+4,4)}$ & $s t$ \\
\hline$E_{(2 t+4,5)}$ & st \\
\hline$E_{(2 t+s+2,2 t+s+2)}$ & $s t(t-1) / 2$ \\
\hline$E_{(2 t+s+2, s+3 t+1)}$ & st \\
\hline$E_{(3 s+t-1,2 t+s+2)}$ & $s t$ \\
\hline$E_{(3 s+t-1, s+3 t+1)}$ & $s$ \\
\hline$E_{(2 t+s+2,2 s+2 t)}$ & $s t(s-1)$ \\
\hline$E_{(2 s+2 t, 2 s+2 t)}$ & $(s(s-1)(s-2)) / 2$ \\
\hline$E_{(3 s+t-1,2 s+2 t)}$ & $s(s-1)$ \\
\hline$E_{(s+3 t+1,2 s+2 t)}$ & $s(s-1)$ \\
\hline$E_{(2 s+2 t, 2 s+2 t)}$ & $s(s-1)$ \\
\hline$E_{(3 s+t-1,3 s+t-1)}$ & $s(s-1) / 2$ \\
\hline$E_{(2 s+2 t, 3 s+t-1)}$ & $s(s-1)$ \\
\hline$E_{(2 s+2 t, 2 s+2 t)}$ & $s-1$ \\
\hline$E_{(2 t+4, s+3 t+1)}$ & $s t$ \\
\hline$E_{(2 t+4,2 s+2 t)}$ & st \\
\hline$E_{(3 t+3,3 t+3)}$ & $s t(t-1) / 2$ \\
\hline$E_{(3 t+3, s+3 t+1)}$ & st \\
\hline$E_{(3 t+3,2 s+2 t)}$ & $s t$ \\
\hline$E_{(s+3 t+1,2 s+2 t)}$ & $s$ \\
\hline
\end{tabular}

$$
\begin{aligned}
M_{1}(H, u)= & \sum_{m l \in E(H)} u^{d_{m}+d_{l}} \\
& +s t u^{(t+5)+(3 t+3)}+s t(s-1) u^{(t+5)+(2 t+4)}+s t(s-1) u^{(t+4)+(2 t+4)}+s t u^{(t+4)+(3 t+3)} \\
& +s t u^{(t+4)+(2 t+s+2)}+s t u^{(t+4)+(5)}+s t u^{(t+4)+(3)}+s t u^{(t+5)+(5)}+s t u^{(5)+(3)} \\
& +s t u^{(2 t+s+3)+(3 t+3)}+s t(t-1) u^{(3 t+3)+(2 t+4)}+s t(t-1) u^{(2 t+s+2)+(2 t+4)} \\
& +s u^{(s+3 t+1)+(2 s+2 t)}+\frac{s t(t-1)(t-2)}{2} u^{(2 t+4)+(2 t+4)}+s t u^{(4)+(3)}+s t u^{(4)+(5)} \\
& +s t u^{(4)+(t+5)}+s t u^{(2 t+4)+(4)}+s t u^{(2 t+4)+(5)}+s t u^{(2 t+s+2)+(s+3 t+1)}+\frac{s t(t-1)}{2} u^{(2 t+s+2)+(2 t+s+2)} \\
& +s t u^{(3 s+t-1)+(2 t+s+2)}+s u^{(3 s+t-1)+(s+3 t+1)}+s t(s-1) u^{(2 t+s+2)+(2 s+2 t)} \\
& +\frac{s(s-1)(s-2)}{2} u^{(2 s+2 t)+(2 s+2 t)}+s(s-1) u^{(3 s+t-1)+(2 s+2 t)}
\end{aligned}
$$




$$
\begin{aligned}
& +s(s-1) u^{(s+3 t+1)+(2 s+2 t)}+s(s-1) u^{(2 s+2 t)+(2 s+2 t)}+\frac{s(s-1)}{2} u^{(3 s+t-1)+(3 s+t-1)} \\
& +s(s-1) u^{(2 s+2 t)+(3 s+t-1)}+(s-1) u^{(2 s+2 t)+(2 s+2 t)}+s t u^{(2 t+4)+(s+3 t+1)} \\
& +s t u^{(2 t+4)+(2 s+2 t)}+\frac{s t(t-1)}{2} u^{(3 t+3)+(3 t+3)}+s t u^{(3 t+3)+(s+3 t+1)}+s t u^{(3 t+3)+(2 s+2 t)}
\end{aligned}
$$

$$
\begin{aligned}
& M_{2}(H, u)=\sum_{m l \in E(H)} u^{d_{m} d_{l}} \\
& +s t u^{(t+5) \times(3 t+3)}+s t(s-1) u^{(t+5) \times(2 t+4)}+s t(s-1) u^{(t+4) \times(2 t+4)} \\
& +s t u^{(t+4) \times(3 t+3)}+s t u^{(t+4) \times(2 t+s+2)}+s t u^{(t+4) \times(5)}+s t u^{(t+4) \times(3)} \\
& +s t u^{(t+5) \times(5)}+s t u^{(5) \times(3)}+s t u^{(2 t+s+3) \times(3 t+3)}+s t(t-1) u^{(3 t+3) \times(2 t+4)} \\
& +s t(t-1) u^{(2 t+s+2) \times(2 t+4)}+\frac{s t(t-1)(t-2)}{2} u^{(2 t+4) \times(2 t+4)}+s t u^{(4) \times(3)} \\
& +s t u^{(4) \times(5)}+s t u^{(4) \times(t+5)}+s t u^{(2 t+4) \times(4)}+s t u^{(2 t+4) \times(5)}+s t u^{(2 t+s+2) \times(s+3 t+1)} \\
& +\frac{s t(t-1)}{2} u^{(2 t+s+2) \times(2 t+s+2)}+s t u^{(3 s+t-1) \times(2 t+s+2)}+s u^{(3 s+t-1) \times(s+3 t+1)} \\
& +s t(s-1) u^{(2 t+s+2) \times(2 s+2 t)}+\frac{s(s-1)(s-2)}{2} u^{(2 s+2 t) \times(2 s+2 t)} \\
& +s(s-1) u^{(3 s+t-1) \times(2 s+2 t)}+s(s-1) u^{(s+3 t+1) \times(2 s+2 t)}+s(s-1) u^{(2 s+2 t) \times(2 s+2 t)} \\
& +\frac{s(s-1)}{2} u^{(3 s+t-1) \times(3 s+t-1)}+s(s-1) u^{(2 s+2 t) \times(3 s+t-1)}+(s-1) u^{(2 s+2 t) \times(2 s+2 t)} \\
& +s t u^{(2 t+4) \times(s+3 t+1)}+s t u^{(2 t+4) \times(2 s+2 t)}+\frac{s t(t-1)}{2} u^{(3 t+3) \times(3 t+3)} \\
& +s t u^{(3 t+3) \times(s+3 t+1)}+s t u^{(3 t+3) \times(2 s+2 t)}+s u^{(s+3 t+1) \times(2 s+2 t)}, \\
& M_{3}(H, u)=\sum_{m l \in E(H)} u^{\left|d_{m}-d_{l}\right|} \\
& +s t u^{(t+5)-(3 t+3)}+s t(s-1) u^{(t+5)-(2 t+4)}+s t(s-1) u^{(t+4)-(2 t+4)}+s t u^{(t+4)-(3 t+3)} \\
& +s t u^{(t+4)-(2 t+s+2)}+s t u^{(t+4)-(5)}+s t u^{(t+4)-(3)}+s t u^{(t+5)-(5)}+s t u^{(5)-(3)} \\
& +s t u^{(2 t+s+3)-(3 t+3)}+s t(t-1) u^{(3 t+3)-(2 t+4)}+s t(t-1) u^{(2 t+s+2)-(2 t+4)}+\frac{s t(t-1)(t-2)}{2} \\
& \cdot u^{(2 t+4)-(2 t+4)}+s t u^{(4)-(3)}+s t u^{(4)-(5)}+s t u^{(4)-(t+5)}+s t u^{(2 t+4)-(4)}+s t u^{(2 t+4)-(5)} \\
& +s t u^{(2 t+s+2)-(s+3 t+1)}+\frac{s t(t-1)}{2} u^{(2 t+s+2)-(2 t+s+2)}+s t u^{(3 s+t-1)-(2 t+s+2)} \\
& +s u^{(3 s+t-1)-(s+3 t+1)}+s t(s-1) u^{(2 t+s+2)-(2 s+2 t)}+\frac{s(s-1)(s-2)}{2} u^{(2 s+2 t)-(2 s+2 t)} \\
& +s(s-1) u^{(3 s+t-1)-(2 s+2 t)}+s(s-1) u^{(s+3 t+1)-(2 s+2 t)}+s(s-1) u^{(2 s+2 t)-(2 s+2 t)} \\
& +\frac{s(s-1)}{2} u^{(3 s+t-1)-(3 s+t-1)}+s(s-1) u^{(2 s+2 t)-(3 s+t-1)}+(s-1) u^{(2 s+2 t)-(2 s+2 t)} \\
& +s t u^{(2 t+4)-(s+3 t+1)}+s t u^{(2 t+4)-(2 s+2 t)}+\frac{s t(t-1)}{2} u^{(3 t+3)-(3 t+3)}+s t u^{(3 t+3)-(s+3 t+1)} \\
& +s t u^{(3 t+3)-(2 s+2 t)}+s u^{(s+3 t+1)-(2 s+2 t)} \text {. }
\end{aligned}
$$


Theorem 5. Let $H$ be the line graph of total of Kragujevac tree; $K \in K g_{q=s(2 t+1)+1, s}$, then we have

$$
\begin{aligned}
& H M(H)=74 s t^{4}+248 s t^{3}+522 s t^{2}+830 s t+173 s^{2} t+293 s^{2} t^{2}+50 s^{2} t^{3} \\
& +36 s^{3} t^{2}+8 s t^{5}-8 s+133 s^{3} t-115 s^{3}+26 s^{2}, \\
& \operatorname{Re} M_{1}(H)=s t^{2}\left[\frac{2}{t^{2}+9 t+20}+\frac{3}{2 t^{2}+14 t+20}+\frac{3}{2 t^{2}+s t+12 t+5 t+14}+\frac{4}{2 t^{2}+s t+12 t+5 s+14}\right. \\
& -\frac{3}{2 t^{2}+14 t+20}+\frac{3}{2 t^{2}+12 t+16}+\frac{4}{3 t^{2}+15 t+12}+\frac{1}{5 t+25}+\frac{5}{6 t^{2}+12 t+3 r t+3 s+6} \\
& +\frac{7}{6 t^{2}+18 t+12}-\frac{5}{6 t^{2}+18 t+12}+\frac{3}{2 t^{2}+6 t+s t+2 s+4}-\frac{2}{2 t^{2}+6 t+s t+2 t+4} \\
& -\frac{1}{4 t^{2}+12 t+2 s t+4 s+8}+\frac{1}{4 t+20}+\frac{1}{4 t+8}+\frac{1}{5 t+10}+\frac{5}{6 t^{2}+6 t+s^{2}+16 s t+3 s} \\
& +\frac{2}{4 t^{2}+4 s t+8 t+s^{2}+8 s+4}-\frac{2}{4 t^{2}+4 s t+8 t+s^{2}+8 s+4}+\frac{3}{7 s t+3 s^{2}+5 s+2 t^{2}-2} \\
& -\frac{2}{3 s t+2 t^{2}+s^{2}+2 s+2 t}+\frac{5}{2 r t+6 t^{2}+14 t+4 s+4}+\frac{1}{t^{2}+s t+2 t+2 s}+\frac{1}{3 t^{2}+6 t+3} \\
& \left.-\frac{1}{3 t^{2}+6 t+3}+\frac{1}{3 t^{2}+4 t+s+s t+4}+\frac{5}{6 t^{2}+6 s t+6 t+6 s}\right]+s t\left[\frac{9}{t^{2}+9 t+20}\right. \\
& +\frac{9}{2 t^{2}+14 t+2 t}+\frac{7}{2 t^{2}+s t+12 t+5 s+4}+\frac{8}{2 t^{2}+s t+12 t+5 s+4}-\frac{9}{2 t^{2}+14 t+20} \\
& -\frac{4}{t^{2}+6 t+8}+\frac{7}{3 t^{2}+15 t+12}+\frac{2}{t+5}+\frac{8}{15}+\frac{5}{6 t^{2}+12 t+3 s t+3 t+6} \\
& -\frac{7}{6 t^{2}+18 t+12}-\frac{3}{2 t^{2}+6 t+s t+2 s+4}+\frac{7}{12}+\frac{9}{20}+\frac{9}{4 t+20}+\frac{1}{t+2} \\
& +\frac{9}{10 t+20}+\frac{3}{6 s t+6 t^{2}+8 t+s^{2}+3 s}-\frac{2}{4 t^{2}+4 s t+8 t+s^{2}+8 s+4} \\
& +\frac{1}{7 s t+3 s^{2}+5 s+2 t^{2}-2}+\frac{4}{7 s t+3 s^{2}+5 s+2 t^{2}-2}-\frac{1}{3 s t+2 t^{2}+s^{2}+2 t+2 s} \\
& +\frac{1}{s^{2}+t^{2}+2 s t}-\frac{3}{6 s^{2}+2 t^{2}+8 s t-2 s-2 t}-\frac{5}{2 s^{2}+8 s t+2 s+2 t+6 t^{2}} \\
& -\frac{1}{s^{2}+t^{2}+2 s t}-\frac{1}{9 s^{2}+t^{2}+6 s t+1-2 t-6 s}-\frac{3}{6 s^{2}+2 t^{2}+8 s t-2 t-2 s} \\
& +\frac{1}{s^{2}+t^{2}+2 s t}+\frac{5}{6 t^{2}+2 s t+14 t+4 s+4}+\frac{1}{s^{2}+s t+2 t+2 s} \\
& \left.-\frac{1}{3 t^{2}+6 t+3}+\frac{4}{9 t^{2}+12 t+3 t+3 s t+3}+\frac{1}{2 t^{2}+2 s t+2 t+2 s}+\frac{5}{6 t^{2}+2 s^{2}+8 s t+2 t+2 s}\right] \\
& +s^{2} t\left[\frac{1}{2 t^{2}+s t+12 t+5 s+14}+\frac{9}{2 t^{2}+14 t+20}+\frac{4}{t^{2}+6 t+8}+\frac{1}{6 t^{2}+12 t+3 s t+3 t+6}\right.
\end{aligned}
$$




$$
\begin{aligned}
& -\frac{1}{4 t^{2}+12 t+2 s t+4 s+8}+\frac{2}{6 s t+6 t^{2}+8 t+s^{2}+3 s}-\frac{1}{4 t^{2}+4 s t+8 t+s^{2}+8 s+4} \\
& +\frac{4}{7 s t+3 s^{2} 5 s+2 t^{2}-2}+\frac{3}{3 s t+2 t^{2}+s^{2}+2 s+2 t}-\frac{3}{6 s t+4 t^{2}+2 s^{2}+4 s+4 t} \\
& -\frac{3}{2 s^{2}+2 t^{2}+4 s t}+\frac{5}{6 s^{2}+2 t^{2}+8 s t+2 s-2 t}+\frac{1}{2 s^{2}+8 s t+2 s+2 t+6 t^{2}} \\
& +\frac{1}{t^{2}+s^{2}+2 s t}+\frac{3}{9 s^{2}+t^{2}+1+6 s t-2 t-6 s}+\frac{1}{8 s t+6 s^{2}+2 t^{2}-2 s-2 t} \\
& +\frac{1}{2 t^{2}+2 s t+4 t+4 s}+\frac{1}{9 t^{2}+12 t+3 s+3 s t+3}+\frac{1}{2 s t+6 t^{2}+14 t+4 s+4} \\
& \left.+\frac{1}{3 t^{2}+3 s t+3 t+3 s}\right]+s^{2} t^{2}\left[\frac{3}{2 t^{2}+14 t+20}+\frac{3}{2 t^{2}+12 t+16}\right. \\
& \left.+\frac{1}{4 t^{2}+12 t+2 s t+4 s+8}+\frac{1}{4 t^{2}+4 s t+8 t+s^{2}+8 s+4}+\frac{1}{3 s t+2 t^{2}+s^{2}+2 s+2 t}\right] \\
& +s t^{3}\left[\frac{5}{6 t^{2}+18 t+12}+\frac{1}{4 t^{2}+12 t+2 s t+4 s+8}+\frac{1}{4 t^{2}+4 s t+8 t+s^{2}+8 s+4}\right. \\
& \left.+\frac{1}{3 t^{2}+6 t+3}\right]+s^{2}\left[\frac{1}{7 s t+3 s^{2}+5 s+2 t^{2}-2}+\frac{1}{s^{2}+t^{2}+2 s t}-\frac{1}{6 s^{2}+2 t^{2}+8 s t+2 s-2 t}\right. \\
& -\frac{1}{6 s^{2}+2 t^{2}+8 s t-2 s-2 t}+\frac{1}{2 s^{2}+8 s t+2 s+2 t+6 t^{2}}
\end{aligned}
$$

$$
\begin{aligned}
& -\frac{1}{t^{2}+r^{2}+2 s t}-\frac{1}{9 s^{2}+t^{2}+1+6 s t-2 t-6 s}-\frac{3}{9 s^{2}+t^{2}+1+6 s t-2 t-6 s} \\
& -\frac{1}{8 s t+6 s^{2}+2 t^{2}-2 t-2 s}-\frac{1}{8 s t+6 s^{2}+2 t^{2}-2 t-2 s}+\frac{1}{t^{2}+s^{2}+2 s t} \\
& \left.+\frac{3}{6 t^{2}+2 s^{2}+8 s t+2 t+2 s}\right]+s^{3} t\left[\frac{1}{6 s t+4 t^{2}+2 s^{2}+4 s+4 t}+\frac{1}{2 s^{2}+2 t^{2}+4 s t}\right] \\
& +s^{4}\left[\frac{1}{2 s^{2}+2 t^{2}+4 s t}\right]+s^{3}\left[\frac{-3}{2 s^{2}+2 t^{2}+4 s t}+\frac{5}{6 s^{2}+2 t^{2}+8 s-2 s-2 t}\right. \\
& +\frac{3}{2 s^{2}+8 s t+2 s+2 t+6 t^{2}}+\frac{1}{t^{2}+s^{2}+2 s t}+\frac{3}{9 s^{2}+t^{2}+6 s t-2 t-6 s} \\
& \left.+\frac{5}{8 s t+6 s^{2}+2 t^{2}-2 t-2 s}\right]+s\left[\frac{1}{6 s^{2}+2 t^{2}+8 s t-2 s-2 t}-\frac{1}{2 s^{2}+8 s t+2 s+2 t+6 t^{2}}\right. \\
& -\frac{1}{9 s^{2}+t^{2}+1+6 s t-2 t-6 s}+\frac{1}{8 s t+6 s^{2}+2 t^{2}-2 t-2 s}-\frac{1}{s^{2}+t^{2}+2 s t} \\
& \left.+\frac{1}{6 t^{2}+2 s^{2} 8 s t+2 s+2 t}\right]+s\left[\frac{-1}{t^{2}+s^{2}+2 s t}\right],
\end{aligned}
$$

$\operatorname{Re} M_{2}(H)=s t^{3}\left[\frac{1}{2 t+9}+\frac{2}{3 t+9}+\frac{2}{3 t+s+7}+\frac{3}{4 t+8}-\frac{2}{3 t+8}+\frac{3}{4 t+7}+\frac{2}{3 t+s+6}\right.$

$$
\begin{aligned}
& +\frac{6}{5 t+s+5}+\frac{18}{5 t+7}-\frac{6}{5 t+7}+\frac{12}{4 t+s+6}+\frac{2}{t+2}+\frac{1}{2 t+4}-\frac{4}{t+2}+\frac{6}{5 t+2 s+3} \\
& +\frac{2}{2 t+s+2}-\frac{1}{2 t+s+2}+\frac{2}{4 s+3 t+1}-\frac{4}{3 s+4 t+2}+\frac{6}{5 t+s+5}+\frac{2}{2 t+s+2}+\frac{3}{2 t+2} \\
& \left.-\frac{3}{4 t+4}+\frac{9}{6 t+s+4}+\frac{6}{5 t+2 s+3}\right]+s t^{2}\left[\frac{9}{2 t+9}+\frac{14}{3 t+9}+\frac{2}{3 t+s+7}+\frac{12}{2 t+s+7}\right. \\
& +\frac{9}{2 t+4}-\frac{12}{3 t+8}+\frac{15}{4 t+7}+\frac{10}{3 t+s+6}+\frac{5}{t+9}+\frac{3}{t+7}+\frac{5}{t+10}+\frac{12}{5 t+s+5} \\
& +\frac{12}{5 t+7}-\frac{18}{5 t+7}+\frac{8}{4 t+s+6}-\frac{2}{2 t^{2}+6 t+s t+2 s+4}+\frac{2}{t+2}-\frac{4}{t+2}+\frac{4}{t+9}
\end{aligned}
$$




$$
\begin{aligned}
& +\frac{4}{t+4}+\frac{10}{2 t+9}+\frac{8}{5 t+2 s+3}+\frac{1}{2 t+s+2}-\frac{2}{2 t+s+2}+\frac{7}{4 s+4 t}-\frac{4}{3 s+t+2} \\
& -\frac{1}{s+t}-\frac{2}{5 s+3 t-1}-\frac{6}{3 s+5 t+1}-\frac{1}{s+t}-\frac{1}{12 s+4 t-4}-\frac{2}{5 s+3 t-1}+\frac{1}{s+t} \\
& \left.+\frac{14}{5 t+s+5}+\frac{4}{2 t+s+2}+\frac{3}{4 t+4}-\frac{3}{2 t+2}+\frac{12}{6 t+s+4}+\frac{6}{5 t+2 s+3}+\frac{6}{3 s+5 t+1}\right] \\
& +s t\left[\frac{20}{2 t+9}+\frac{20}{3 t+9}+\frac{14}{2 t+s+7}+\frac{15}{4 t+8}-\frac{16}{3 t+8}+\frac{12}{4 t+7}+\frac{8}{3 t+s+6}\right. \\
& +\frac{20}{t+9}+\frac{12}{t+7}+\frac{25}{t+10}+\frac{15}{8}+\frac{6}{5 t+s+5}-\frac{12}{5 t+7}-\frac{3}{2 t^{2}+6 t+s t+2 s+4} \\
& +\frac{2}{t+2}+\frac{12}{7}+\frac{20}{9}+\frac{20}{t+9}+\frac{8}{t+4}+\frac{10}{2 t+9}-\frac{1}{2 t+s+2}-\frac{2}{4 s+3 t+1}+\frac{4}{5 t+s+5} \\
& -\frac{1}{2 s+2 t}+\frac{2}{5 s+3 p-1}-\frac{2}{3 s+5 p+1}-\frac{3}{6 s+2 t-2}+\frac{2}{5 s+3 t-1}-\frac{2}{s+t} \\
& \left.-\frac{3}{2 t+4}+\frac{3}{6 t+s+4}+\frac{2}{3 s+5 t+1}\right]+s^{2} t^{2}\left[\frac{1}{3 t+s+7}+\frac{4}{t+3}+\frac{1}{3 t+s+6}\right. \\
& +\frac{3}{5 t+s+5}+\frac{4}{4 t+s+6}+\frac{6}{3 s+5 t+1}+\frac{1}{s+t}+\frac{1}{12 s+4 t+4}+\frac{2}{5 s+3 t-1} \\
& \left.+\frac{2}{5 t+s+5}+\frac{2}{2 t+s+2}+\frac{6}{5 t+2 s+3}+\frac{3}{6 t+s+4}\right]+s^{2} t\left[\frac{5}{3 t+r+7}\right. \\
& +\frac{16}{3 t+9}+\frac{4}{3 t+s+6}+\frac{3}{5 t+s+5}-\frac{1}{4 t^{2}+12 t+2 s t+4 s+8}+\frac{3}{6 t+s+4} \\
& +\frac{3}{5 t+2 s+3}-\frac{2}{2 t+s+2}+\frac{5}{4 s+3 t+1}+\frac{5}{2 s+2 t}-\frac{4}{3 s+4 t+2}+\frac{2}{s+t} \\
& -\frac{2}{5 s+3 t-1}-\frac{8}{5 s+3 t-1}+\frac{2}{3 s+5 t+1}-\frac{8}{3 s+5 t+1}-\frac{2}{s+t}-\frac{1}{6 s+2 t-2} \\
& +\frac{3}{6 s+2 t-2}-\frac{8}{5 s+3 t-1}-\frac{2}{5 s+3 t-1}+\frac{2}{s+t}+\frac{4}{5 t+s+5}+\frac{4}{2 t+s+2} \\
& \left.+\frac{6}{5 t+2 s+3}+\frac{8}{3 s+5 t+1}\right]+s^{2} t^{3}\left[\frac{2}{3 t+9}+\frac{2}{4 t+s+6}+\frac{1}{2 t+s+2}+\frac{4}{3 s+4 t+2}\right] \\
& +s t^{4}\left[\frac{6}{5 t+7}+\frac{4}{4 t+s+6}+\frac{2}{t+2}+\frac{1}{4 t+4}-\frac{1}{t+2}+\frac{1}{2 t+s+2}\right]+s t^{5}\left[\frac{1}{2 t+4}\right] \\
& +s^{3} t\left[\frac{1}{5 t+2 s+3}-\frac{1}{8 t+4 s+8}+\frac{3}{4 s+3 t+1}+\frac{4}{3 s+4 t+2}-\frac{2}{3 s+4 t+2}-\frac{3}{s+t}\right. \\
& \left.+\frac{8}{5 s+3 t-1}+\frac{8}{3 s+5 t+1}+\frac{2}{s+t}+\frac{3}{6 s+2 t-2}+\frac{8}{5 s+3 t-1}\right]+s^{3} t^{2}\left[\frac{1}{8 t+4 r+8}\right. \\
& \left.+\frac{6}{3 s+4 t+2}+\frac{1}{2 s+2 t}\right]+s^{3}\left[\frac{3}{4 s+4 t}+\frac{1}{s+t}-\frac{2}{5 s+3 t-1}-\frac{6}{5 s+3 t-1}\right. \\
& +\frac{2}{3 s+5 t+1}-\frac{2}{3 s+5 t+1}-\frac{1}{s+t}-\frac{3}{6 s+2 t-2}-\frac{9}{12 s+4 t t-4}-\frac{2}{5 s+3 t-1} \\
& \left.-\frac{6}{5 s+3 t-1}+\frac{1}{s+t}+\frac{2}{3 s+5 t+1}\right]+s^{2}\left[\frac{1}{2 s+2 t}+\frac{2}{5 s+3 t-1}-\frac{2}{3 s+5 t+1}\right. \\
& \left.+\frac{1}{12 s+4 t-4}-\frac{3}{6 s+2 t-2}+\frac{2}{5 s+3 t-1}+\frac{2}{3 s+5 t+1}\right]+s\left[\frac{-1}{4 s+4 t}-\frac{1}{12 s+4 t-4}\right] \\
& +s^{4} t\left[\frac{2}{3 s+4 t+2}+\frac{1}{s+t}\right]+s^{5}\left[\frac{1}{2 s+2 t}\right]+s^{4}\left[\frac{-3}{2 s+2 t}+\frac{6}{5 s+3 t-1}\right.
\end{aligned}
$$




$$
\left.+\frac{2}{3 s+5 t+1}+\frac{1}{s+t}+\frac{9}{12 s+4 t-4}+\frac{6}{5 s+3 t-1}\right]+t^{2}\left[\frac{-1}{s+t}\right]
$$

$\operatorname{Re} M_{3}(H)=s t\left[143 t^{3}+406 t^{2}+1000 t+1745+141 s t^{2}+48 s^{2}+304 s t+128 s+50 s^{2} t+7 s^{3}\right]$

$$
\begin{aligned}
& +s^{2} t\left[28 t^{3}+136 t^{2}+338 t+308+6 s^{3}+26 s^{2} t+36 s t^{2}+16 s^{2}+40 s t+8 s\right]+s t^{2} \\
& \cdot\left[65 t^{3}+213 t^{2}+203 t+39+8 s^{2} t+52 s+14 s^{2}+24 s t^{2}+72 s t+2 s^{3}\right]+s t^{3}\left[8 t^{3}+48 t^{2}\right. \\
& +96 t+64]+s\left[-59 s^{3}+15 t^{3}+9 s^{2} t+67 s^{2}\right]+s\left[29 s t^{2}+11 t^{2}-17 s-11 t+66 s t\right] \\
& +s^{3}\left[8 t^{3}+8 s^{2}+24 s t^{2}+24 s^{2} t\right]+s^{2}\left[5 t^{3}+85 s^{3}+105 s t^{2}\right. \\
& \left.+153 s^{2} t-51 s^{2}-3 t^{2}-4 s t+9 t+15 s-2\right], \\
& { }^{m} M_{1}(H)=s t\left[\frac{1}{2 t+9}+\frac{1}{3 t+9}+\frac{1}{3 t+s+7}+\frac{1}{4 t+8}+\frac{1}{3 t+9}-\frac{1}{3 t+8}+\frac{1}{4 t+7}\right. \\
& +\frac{1}{3 t+s+6}+\frac{1}{t+9}+\frac{1}{t+7}+\frac{1}{t+10}+\frac{1}{8}+\frac{1}{5 t+s+5}-\frac{1}{5 t+7}-\frac{1}{4 t+s+6} \\
& +\frac{1}{2(4 t+8)}+\frac{1}{7}+\frac{1}{9}+\frac{1}{t+9}+\frac{1}{2 t+8}+\frac{1}{2 t+9}+\frac{1}{5 s+2 t+3}-\frac{1}{4 t+2 s+4} \\
& +\frac{1}{4 s+3 t+1}-\frac{1}{3 s+4 t+2}+\frac{1}{5 t+s+5}+\frac{1}{4 t+2 s+4}-\frac{1}{6 t+6}+\frac{1}{6 t+s+4} \\
& \left.+\frac{1}{5 t+2 s+3}\right]+s^{2} t\left[\frac{1}{3 t+9}+\frac{1}{3 t+8}+\frac{1}{5 t+7}+\frac{1}{3 s+4 t+2}\right]+s t^{2}\left[\frac{1}{4 t+s+6}\right. \\
& \left.-\frac{1}{4 t+8}+\frac{1}{2(4 t+2 s+4)}+\frac{1}{6 t+6}\right]+s t^{3}\left[\frac{1}{4 s+4 t}+\frac{1}{4 s+4 t}-\frac{1}{5 s+3 t-1}\right. \\
& \left.-\frac{1}{3 s+5 t+1}-\frac{1}{4 s+4 t}-\frac{1}{2(6 s+2 t+2)}-\frac{1}{5 s+3 t-1}+\frac{1}{4 s+4 t}+\frac{1}{3 s+5 t+1}\right] \\
& +s^{2}\left[\frac{-3}{2(4 s+4 t)}+\frac{1}{5 s+3 t+1}+\frac{1}{3 s+5 t+1}+\frac{1}{4 s+4 t}+\frac{1}{2(6 s+2 t-2)}+\frac{1}{5 t+3 t-1}\right] \\
& +s^{3}\left[\frac{1}{4 s+4 t}\right]-\frac{1}{4 s+4 t}, \\
& R M_{2}(H)=s t\left[\frac{-51}{2} t^{2}+53 t+102+6 s t+11 s+s^{2}\right]+s^{2} t\left[8 t^{2}+20+2 s^{2}+8 s t+s\right]+s t^{2}\left[\frac{25}{2} t^{2}\right. \\
& \left.+17 t-\frac{11}{2}+6 s t+4 s+\frac{s^{2}}{2}\right]+\frac{s t^{3}}{2}\left[4 t^{2}+12 t+9\right]+s\left[\frac{-19 s^{2}}{2}-\frac{11 t^{2}}{2}\right. \\
& -5 s t+30 s+21 t-12]+s^{3}\left[2 s^{2}+2 t^{2}+4 s t-2 s-2 t+\frac{1}{2}\right]+s^{2}\left[\frac{33 s^{2}}{2}+\frac{17 t^{2}}{2}\right. \\
& \left.+17 s t-19 s-13 t+\frac{11}{2}\right], \\
& \operatorname{RRR}(H)=s t\left[\sqrt{t^{2}+12+7 t}+\sqrt{2 t^{2}+11 t+12}+\sqrt{3 t^{2}+14 t+8}+\sqrt{2 t^{2}+9 t+4 s+s t+4}\right. \\
& -\sqrt{2 t^{2}+11 t+12}-\sqrt{2 t^{2}+7 t+3 s+s t+3}+\sqrt{2 t^{2}+9 t+9}+\sqrt{3 t^{2}+11 t+6} \\
& +\sqrt{4 t+12}+\sqrt{2 t+6}+\sqrt{4 t+16}+\sqrt{8}+\sqrt{6 t^{2}+7 t+2 s+3 s t+2}-\sqrt{6 t^{2}+13 t+6} \\
& -\sqrt{4 t^{2}+8 t+3 s+4 s t+1}+\frac{1 \sqrt{4 t^{2}+12 t+(9 / 2)}}{2}+\sqrt{6}+\sqrt{12}
\end{aligned}
$$




$$
\begin{aligned}
& +\sqrt{3 t+12}+\sqrt{6 t+9}+\sqrt{8 t+12}+\sqrt{6 t^{2}+5 s t+s^{2}+3 t+s} \\
& \mathrm{GO}_{1}(H)=s t\left[\frac{111 t^{2}}{2}+184 t+\frac{603}{2}+55 s+23 s t+2 s^{2}\right]+s^{2} t\left[6 t^{2}+40 t+55+6 s t+3 s^{2}+4 s\right]+s t^{2} \\
& \cdot\left[\frac{29 t^{2}}{2}+51 t+\frac{81}{2}+5 s+2 s t\right]+s\left[\frac{5 t^{2}}{2}-\frac{19 s^{2}}{2}-4 s-2 t-s t+\frac{3}{2}\right]+s^{3}\left[2 s+2 t+2 s^{2}\right. \\
& \left.+2 t^{2}+4 s t\right]+s^{2}\left[\frac{17 t^{2}}{2}+\frac{33 s^{2}}{2}+9 s+7 t+23 s t-\frac{1}{2}\right]-\left[4 t+4 s+14 t^{2}+4 s^{2}+8 s t\right] \\
& \mathrm{GO}_{2}(H)=s t\left[143 t^{3}+406 t^{2}+1000 t+1745+141 s t^{2}+48 s^{2}+304 s t+128 s+50 s^{2} t+7 s^{3}\right]+s^{2} t \\
& \cdot\left[28 t^{3}+136 t^{2}+338 t+308+6 s^{3}+26 s^{2} t+36 s t^{2}+16 s^{2}+40 s t+8 s\right]+s t^{2}\left[65 t^{3}+213 t^{2}\right. \\
& \left.+203 t+39+8 s^{2} t+52 s+14 s^{2}+24 s t^{2}+72 s t+2 s^{3}\right]+s t^{3}\left[8 t^{3}+48 t^{2}+96 t+64\right] \\
& +s\left[-59 s^{3}+15 t^{3}+9 s^{2} t+67 s^{2}+29 s t^{2}+11 t^{2}-17 s-11 t+66 s t\right]+s^{3}\left[8 t^{3}+8 s^{2}\right. \\
& \left.+24 s t^{2}+24 s^{2} p\right]+s^{2}\left[5 t^{3}+85 s^{3}+105 s t^{2}+153 s^{2} t-51-s^{2}-3 t^{2}-4 s t+9 t+15 s-2\right], \\
& \mathrm{HGO}_{1}(H)=s t\left[\frac{783 t^{4}}{2}+1851 t^{2}+1114 t^{3}+1148 s t^{2}+320 s^{2} t+9596 t+126 s^{2} t^{2}+354 s t^{3}\right. \\
& \left.+441 s^{2}+10 s^{4}+1855 s t+\frac{13611}{1}+654 s+12 s^{3} t+59 s^{3}\right]+s^{2} t\left[24 t^{4}\right. \\
& +734 t^{2}+192 t^{3}+4 s^{4}+28 s^{3}+16 s^{2} t^{2}+56 s t^{2}+32 s^{2} t+57 s^{2}+112 s t \\
& +1738 t+28 s+1421]+s t^{2}\left[\frac{185 t^{4}}{2}+629 t^{3}+1548 t^{2}+16 s t^{3}+104 s t^{2}+20 s^{2} t\right. \\
& \left.+4 s^{2} t^{2}+25 s^{2}+216 s t+140 s+1622 t+\frac{1339}{2}\right]+s^{3}\left[8 t^{4}+8 s^{4}+24 s^{2} t^{2}+8 s^{2}\right. \\
& \left.+8 t^{2}+48 s^{3} t+16 s t+48 s t^{3}+16 s^{3}+24 t^{3}+48 s^{2} t+56 s t^{2}\right]+s^{2}\left[36 t^{4}+109 s^{4}\right. \\
& +68 t^{3}+40 s^{3}+224 s^{3} t+118 s t^{3}+267 s^{2} t^{2}+110 s^{2} t+191 s t^{2}-s^{2}+46 t^{2}+138 s t \\
& \left.+10 t+4 s+\frac{7}{2}\right]+s\left[-108 s^{4}+t^{4}-212 s^{3} t-10 s t^{3}-109 s^{2} t^{2}-20 s^{3}+4 t^{3}+48 s^{2}\right. \\
& +\left[3 t^{2}+126 s^{2} t+s t^{2}+10 t-6 s+23 s t-\frac{3}{2}-\left[16 t^{4}+14 s^{4}+48 s^{2} t^{2}+16 s^{2}+16 t^{2}\right.\right. \\
& \left.+96 s^{3} t+72 s t+96 s t^{3}+32 s^{3}+48 t^{3}+96 s^{2} t+112 s t^{2}\right] \\
& \mathrm{HGO}_{2}(H)=s t\left[\left(2 t^{3}+27 t^{2}+121 t+180\right)^{2}+\left(6 t^{3}+60 t^{2}+180 t+180\right)^{2}+\left(6 t^{3}+5 s t^{2}+5 s^{2}+50 t^{2}\right.\right. \\
& \left.+34 s t+126 t+98+49 t+s^{2} t\right)^{2}+\left(12 t^{3}+96 t^{2}+204 t+120\right)^{2}-\left(6 t^{3}+60 t^{2}+18 t+180\right)^{2}- \\
& \cdot\left(6 t^{3}+52 t^{2}+144 t+128\right)^{2}+\left(12 t^{3}+81 t^{2}+153 t+84\right)^{2}+\left(5 t^{2}+65 t+180\right)^{2}+\left(20 t^{2}+130 t\right. \\
& +180)^{2}+\left(5 t^{2}+25 t+250\right)^{2}+\left(6 t^{3}-5 s t^{2}+s^{2} t+48+42 t^{2}+28 s t+84 t+4 s^{2}+32 s\right)^{2} \\
& +\left(3 t^{2}+33 t+84\right)^{2}+14400+\left(30 t^{3}+90 t^{2}+3 s^{2}+3 s^{2} t+21 s t^{2}+30+42 s t+40 t+21 s\right)^{2} \\
& -\left(30 t^{3}+132 t^{2}+185 t+84\right)^{2}-\left(16 t^{3}+2 s^{2} t+32 s+72 t^{2}+4 s^{2}+48+104 t+12 s t^{2}+40 s t\right)^{2} \\
& +\frac{1}{2}\left(16 t^{3}+96 t^{2}+192 t+128\right)^{2}+7056+32400+\left(4 t^{2} 50 t+180\right)^{2}+\left(16 t^{2}+96 t+128\right)^{2} \\
& \operatorname{PGO}(H)=s t\left[\frac{1}{\sqrt{2 t^{3}+27 t^{2}+121 t+180}}+\frac{1}{\sqrt{6 t^{3}+60 t^{2}+186 t+180}}\right.
\end{aligned}
$$




$$
\begin{aligned}
& +\frac{1}{\sqrt{12 t^{3}+96 t^{2}+204 s+120}}-\frac{1}{\sqrt{6 t^{3}+169 t^{2}+186 t+180}}+\frac{1}{\sqrt{5 t^{2}+65 t+180}} \\
& -\frac{1}{\sqrt{6 t^{3}+52 t^{2}+144 t+128}}+\frac{1}{\sqrt{6 t^{3}+5 s t^{2}+5 s^{2}+50 t^{2}+34 s t+126 t+98+49 s+s^{2} t}} \\
& +\frac{1}{\sqrt{12 t^{3}+81 t^{2}+153 t+84}}+\frac{1}{\sqrt{6 t^{3}-5 s t^{2}+s^{2} t+48+42 t^{2}+28 s t+84 t+4 s^{2}+32 s}} \\
& +\frac{1}{\sqrt{5 t^{2}+75 t+250}}+\frac{1}{\sqrt{120}}+\frac{1}{\sqrt{30 t^{3}+90 t^{2}+3 s^{2}+3 s^{2} t+21 s t^{2}+30+42 s t+90 t+121 s}} \\
& -\frac{1}{\sqrt{30 t^{3}+132 t^{2}+186 t+84}}+\frac{1}{\sqrt{84}} \\
& -\frac{1}{\sqrt{16 t^{3}+2 s^{2} t+32 s+72 s^{2}+4 s^{2}+48+104 t+112 s t^{2}+40 s t}} \\
& +\frac{1}{2 \sqrt{16 t^{3}+96 t^{2}+192 t+128}}+\frac{1}{\sqrt{180}}+\frac{1}{\sqrt{4 t^{2}+50 t+180}}+\frac{1}{\sqrt{16 t^{2}+96 t+128}} \\
& +\frac{1}{\sqrt{30 t^{3}+2 s^{3}+9 s+34 t+9 s^{2}+58 t^{2}+17 s^{2} t+49 s t+42 s t^{2}}}+\frac{1}{\sqrt{3 t^{2}+33 t+84}} \\
& -\frac{1}{2 \sqrt{16 t^{3}+2 s^{3}+24 s t^{2}+16+48 t^{2}+12 s^{2} t+64 s t+48 t+20 s^{2}+40 s}} \\
& +\frac{1}{\sqrt{12 s^{3}+6 t^{3}+37 s^{2} t-6 t+23 s^{2}+29 s t^{2}-3 s+22 s t+2 t^{2}-2}} \\
& -\frac{1}{\sqrt{6 s^{3}+16 t^{3}+26 s^{2} t+36 s t^{2}+16 s^{2}+40 s t+24 t^{2}+8 s+8 t}} \\
& +\frac{1}{\sqrt{3 t^{3}+44 r t+16 s t^{2}+2 s^{2} t+4 s^{2}+20+100 t^{2}+90 t+24 s}} \\
& +\frac{1}{\sqrt{16 t^{3}+24 s t^{2}+48 t^{2}+64 s t+8 s^{2} t+16 s^{2}+32 t+32 s}} \\
& -\frac{1}{2 \sqrt{54 t^{3}+162 t^{2}+16 t+54}}+\frac{1}{\sqrt{64 t^{3}+3 s^{2}+108 t^{2}+42 s t+27 s t^{2}+12+66 t+3 s^{2} t+15 s}} \\
& \left.+\frac{1}{\sqrt{30 t^{3}+12 s^{2} t+12 s^{2}+18 t+18 s+48 t^{2}+142 s t^{2}+60 s t}}\right]+s^{2} t\left[\frac{1}{\sqrt{6 t^{3}+60 t^{2}+186 t+180}}\right. \\
& \left.+\frac{1}{\sqrt{6 t^{3}+52 t^{2}+144 t+128}}+\frac{1}{\sqrt{6 t^{3}+16 t^{3}+26 s^{2} t+136 s t^{2}+16 s^{2}+40 s t+24 t^{2}+8 s+8 t}}\right] \\
& +s t^{2}\left[\frac{1}{\sqrt{30 t^{3}+132 t^{2}+186 t+84}}-\frac{1}{\sqrt{16 t^{3}+96 t^{2}+192 t+128}}\right. \\
& +\frac{1}{\sqrt{16 t^{3}+2 s^{2} t+32 s+12 t^{2}+4 s^{2}+48+104 s+112 s t^{2}+40 s t}} \\
& +\frac{1}{2 \sqrt{16 t^{3}+2 s^{3}+24 s t^{2}+16+48 t^{2}+12 s^{2} t+64 s t+48 t+20 s^{2}+40 s}} \\
& \left.+\frac{1}{2 \sqrt{54 t^{3}+162 t^{2}+162 t+54}}\right]+s t^{3}\left[\frac{1}{2 \sqrt{16 t^{3}+9 s^{2}+182 t+128}}\right] \\
& +s\left[\frac{1}{\sqrt{12 s^{3}+12 t^{3}+52 s^{2} t+8 s^{2}-8 t^{2}+52 s t^{2}-4 s-4 t}}+\frac{1}{\sqrt{16 t^{3}+16 s^{3}+48 s t^{2}+48 s^{2} t}}\right.
\end{aligned}
$$




$$
\begin{aligned}
& -\frac{1}{\sqrt{30 s^{3}+6 t^{3}+2 t+2 s+34 s t^{2}+58 s^{2} t-16 s^{2}-24 s t-8 t^{2}}} \\
& -\frac{1}{\sqrt{6 s^{3}+30 t^{3}+16 t^{2}+34 s^{2} t+2 t+2 s+8 t^{2}+24 s t+52}} \\
& -\frac{1}{2 \sqrt{54 s^{3}+2 t^{3}+18 s t^{2}+18 s+54 s^{2} t-36 s t-54 s^{2}+6 t-6 t^{2}-2}} \\
& -\frac{1}{\sqrt{30 s^{3}+6 t^{3}+2 t+2 s+34 s t^{2}+58 s^{2} t-16 s^{2}-24 s t-8 t^{2}}} \\
& \left.+\frac{1}{\sqrt{16 t^{3}+16 s^{3}+48 s t^{2}+48 s^{2} t}}+\frac{1}{\sqrt{58 s t^{2}+3 t^{3}+6 s^{3}+34 s t^{2} t+2 s+2 t+24 s t+8 s^{2}+16 t^{2}}}\right] \\
& +s^{3}\left[\frac{1}{2 \sqrt{16 t^{3}+16 s^{3}+48 s t^{2}+48 s^{2} t}}\right]+s^{2}\left[\frac{1}{\sqrt{30 s^{3}+6 t^{3}+2 t+2 s+34 s t^{2}+58 s^{2} t-16 s^{2}-24 s t-8 t^{2}}}\right. \\
& -\frac{3}{2 \sqrt{16 t^{3}+16 s^{3}+48 s t^{2}+48 s^{2} t}}+\frac{1}{\sqrt{6 s^{3}+30 t^{3}+16 t^{2}+34 s^{2} t+2 t+2 s+8 t^{2}+24 s t+52 s t^{2}}} \\
& +\frac{1}{2 \sqrt{54 s^{3}+2 t^{3}+18 s t^{2}+18 s+54 s^{2} t-36 s t-54 s^{2}+6 t-6 t^{2}-2}} \\
& +\frac{1}{\sqrt{30 s^{3}+6 t^{3}+2 t+2 s+34 s t^{2}+58 s^{2} t-16 s^{2}-24 s t-8 t^{2}}} \\
& \left.+\frac{1}{\sqrt{16 t^{3}+16 s^{3}+48 s t^{2}+48 s^{2} t}}\right]-\left[\frac{1}{\sqrt{16 t^{3}+16 s^{3}+48 s t^{2}+48 s^{2} t}}\right] \text {, } \\
& \operatorname{SGO}(H)=s t\left[\frac{1}{\sqrt{t^{2}+11 t+29}}+\frac{1}{\sqrt{2 t^{2}+17 t+29}}+\frac{1}{\sqrt{2 t^{2}+15 t+6 s+s t+21}}+\frac{1}{\sqrt{3 t^{2}+22 t+23}}\right. \\
& -\frac{1}{\sqrt{2 t^{2}+17 t+29}}-\frac{1}{\sqrt{2 t^{2}+15 t+24}}+\frac{1}{\sqrt{3 t^{2}+20 t+29}}+\frac{1}{\sqrt{6 t+35}}+\frac{1}{\sqrt{6 t+35}} \\
& +\frac{1}{\sqrt{23}}+\frac{1}{\sqrt{16 t^{2}+17 t+9 s+3 s t+1}}-\frac{1}{\sqrt{6 t^{2}+23 t+29}}-\frac{1}{\sqrt{4 t^{2}+20 t+24}} \\
& +\frac{1}{\sqrt{19}}+\frac{1}{\sqrt{29}}+\frac{1}{\sqrt{5 t+29}}+\frac{1}{\sqrt{10 t+29}}+\frac{1}{\sqrt{6 t^{2}+2 s^{2}+13 t+5 s+6 s t+3}} \\
& +\frac{1}{\sqrt{3 s^{2}+2 t^{2}+3 t+9 s+1}}-\frac{1}{\sqrt{4 t^{2}+2 s^{2}+6 s t+8 t+7 s+2}}+\frac{1}{\sqrt{6 t^{2}+2 s t+19 s+15 t+9}} \\
& +\frac{1}{\sqrt{4 t^{2}+4 s t+10 s+12 t+4}}-\frac{1}{2 \sqrt{9 t^{2}+24 t+15}}+\frac{1}{\sqrt{9 t^{2}+18 t+4 s+3 s t+7}} \\
& \left.+\frac{1}{\sqrt{6 t^{2}+6 s t+8 s+11 t+13}}\right]+s^{2} t\left[\frac{1}{\sqrt{2 t^{2}+17 t 29}}+\frac{1}{\sqrt{2 t^{2}+15 t+29}}\right. \\
& \left.+\frac{1}{\sqrt{4 t^{2}+2 s^{2}+7 s+8 t+2}}\right]+s t^{2}\left[\frac{1}{\sqrt{6 t^{2}+23 t+9}}+\frac{1}{\sqrt{4 t^{2}+2 s t+5 s+16 t+14}}\right. \\
& \left.+\frac{1}{2 \sqrt{9 t^{2}+24 t+15}}\right]+s\left[\frac{1}{\sqrt{3 s+3 t^{2}+10 s t+2 t+6 s-1}}+\frac{1}{\sqrt{4 s^{2}+4 t^{2}+8 s t+4 s+4 t}}\right. \\
& -\frac{1}{\sqrt{6 s^{2}+2 t^{2}+8 s t+3 s+t-1}}-\frac{1}{\sqrt{4 s^{2}+4 t^{2}+8 s t+4 t+4 s}}-\frac{1}{2 \sqrt{9 s^{2}+t^{2}+6 s t-1}} \\
& \left.-\frac{1}{\sqrt{6 s^{2}+2 t^{2}+8 s t+3 s+t-1}}+\frac{1}{\sqrt{6 t^{2}+8 s t+7 t+5 s+1}}\right]+s^{3} \\
& \cdot\left[\frac{1}{2 \sqrt{4 s^{2}+4 t^{2}+8 s t+4 s+4 t}}\right]+s^{2}\left[\frac{1}{\sqrt{6 s^{2}+2 t^{2}+8 s t+t+3 s-1}}-\right. \\
& \cdot \frac{3}{2 \sqrt{4 s^{2}+4 t^{2}+8 s t+4 s+4 t}}+\frac{1}{\sqrt{4 s^{2}+4 t^{2}+8 s t+4 s+4 t}}
\end{aligned}
$$




$$
\begin{aligned}
+ & \left.\frac{1}{\sqrt{2 s^{2}+6 t^{2}+8 s t+7 s+5 t}}+\frac{1}{2 \sqrt{9 s^{2}+6 s t+t^{2}-1}}+\frac{1}{\sqrt{6 s^{2}+2 t^{2}+8 s t+3 s+t-1}}\right] \\
& -\left[\frac{1}{\sqrt{4 s^{2}+4 t^{2}+8 s t+4 s+4 t}}\right], \\
F(H)= & s t\left[84 t^{2}+18 s^{2}+40 s t+263 t+8 s+476\right]+s^{2} t\left[18 t^{2}+5 s^{2}+12 s t+58 t+4 s+77\right] \\
& +s^{2} t\left[26 t^{2}+2 s^{2}+8 s t+52 t+8 s+26\right]+s t^{2}\left[4 t^{2}+16+16 t\right]+s^{3}\left[4 t^{2}+4 s^{2}+8 s t\right] \\
& +s\left[-17 s^{2}+7 t^{2}-6 s t+10 t+14 s-1\right]+s^{2}\left[20 t^{2}+36 s^{2}+40 s t-16 s+4\right] \\
& -\left[8 t^{2}+8 s^{2}+16 s t\right] .
\end{aligned}
$$

Proof. For line distribution shown in Table 2, use of formulas (5)-(9), (11)-(18), and (22) will give desired above expressions.
Theorem 6. Let $H$ be the line graph of total of Kragujevac tree; $K \in K g_{q=s(2 t+1)+1, s}$, then M-polynomial and certain topological indices that can be deducted from M-polynomial are

$$
\begin{aligned}
& M(H ; u, v)=s t u^{t+5} v^{t+4}+s t u^{2 t+4} v^{t+5}+s t u^{t+5} v^{2 t+s+2}+s t u^{t+5} v^{3 t+3}+s t(s-1) u^{t+5} v^{2 t+4} \\
& +s t(s-1) u^{t+4} v^{2 t+4}+s t u^{t+4} v^{3 t+3}+s t u^{t+4} v^{2 t+s+2}+s t u^{t+4} v^{5}+s t u^{t+4} v^{3}+s t u^{t+5} \\
& \cdot v^{5}+s t u^{5} v^{3}+s t u^{2 t+s+2} v^{3 t+3}+s t(s-1) u^{3 t+3} v^{2 t+4}+s t(s-1) u^{2 t+s+2} v^{2 t+4} \\
& +\frac{s t(t-1)(t-2)}{2} u^{2 t+4} v^{2 t+4}+s t u^{4} v^{3}+s t u^{4} v^{5}+s t u^{4} v^{t+5}+s t u^{2 t+4} v^{4}+s t u^{2 t+4} v^{5} \\
& +s t u^{2 t+s+2} v^{s+3 t+1}+\frac{s t(t-1)}{2} u^{2 t+s+2} v^{2 t+s+2}+s t u^{3 s+t-1} v^{2 t+s+2} \\
& +s u^{3 s+t-1} v^{s+3 t-1}+s t(s-1) u^{2 t+s+2} v^{2(s+t)}+\frac{s(s-1)(s-2)}{2} u^{2(s+t)} v^{2(s+t)} \\
& +s(s-1) u^{3 s+t-1} v^{2(s+t)}+s(s-1) u^{s+3 t+1} v^{2(s+t)}+\frac{s(s-1)}{2} u^{3 s+t-1} v^{3 s+t-1} \\
& +s(s-1) u^{2 t+2 s} v^{3 s+t-1}+(s-1) u^{2 t+2 s} v^{2 t+2 s}+s t u^{2 t+4} v^{s+3 t+1}+s t u^{2 t+4} v^{2 t+2 s} \\
& +\frac{s t(t-1)}{2} u^{3 t+3} v^{3 t+3}+s t u^{3 t+3} v^{s+3 t+1}+s t u^{3 t+3} v^{2 t+2 s} \\
& +s u^{s+3 t+1} v^{2 t+2 s}+s(s-1) u^{2 t+2 s} v^{2(t+s)}, \\
& M_{1}(H)=s t(t+5)+s t(2 t+4)+s t(t+5)+s t(t+5)+s t(s-1)(t+5)+s t(s-1)(t+4) \\
& +s t(t+4)+s t(t+4)+s t(t+5)+s t(5)+s t(2 t+s+2)+s t(s-1)(3 t+3)+s t(s-1) \\
& \cdot(2 t+s+2)+\frac{s t(t-1)(t-2)}{2}(2 t+4)+s t(4)+s t(4)+s t(4)+s t(2 t+4) \\
& +s t(2 t+4)+s t(2 t+s+2)+\frac{s t(t-1)}{2}(2 t+s+2)+s t(3 s+t-1)+s(3 s+t-1) \\
& +s t(s-1)(2 t+s+2)+\frac{s(s-1)(s-2)}{2}(2 s+2 t)+s(s-1)(3 s+t-1)+s(s-1) \\
& \cdot(s+3 t+1)+s(s-1)(2 t+2 s)+\frac{s(s-1)}{2}(3 s+t-1)+s(s-1)(2 t+2 s)+(s-1)
\end{aligned}
$$




$$
\begin{aligned}
& \cdot(2 t+2 s)+s t(2 t+4)+s t(2 t+4)+\frac{s t(t-1)}{2}(3 t+3)+s t(3 t+3)+s t(3 t+3) \\
& +s(s+3 t+1)+s t(t+4)+s t(t+5)+s t(2 t+s+2)+s t(3 t+3)+s t(s-1)(2 t+4) \\
& +s t(s-1)(2 t+4)+s t(3 t+3)+s t(2 t+s+2)+s t(t+4)+s t(t+4)+s t(5)+s t(3) \\
& +s t(5)+s t(3)+s t(3 t+3)+s t(s-1)(2 t+4)+s t(s-1)(2 t+4)+\frac{s t(t-1)(t-2)}{2} \\
& \cdot(2 t+4)+s t(3)+s t(5)+s t(t+5)+s t(4)+s t(5)+s t(s+3 t+1) \\
& +\frac{s t(t-1)}{2}(2 t+s+2)+s t(2 t+s+2)+s(s+3 t-1)+s t(s-1)(2 t+2 s) \\
& +\frac{s(s-1)(s-2)}{2}(2 t+2 s)+s(s-1)(2 t+2 s)+s(s-1)(2 t+2 s)+s(s-1)(2 t+2 s) \\
& +\frac{s(s-1)}{2}(3 s+t-1)+s(s-1)(3 s+t-1)+(s-1)(2 t+2 s)+s t(s+3 t+1)+s t(2 t+2 s) \\
& +\frac{s t(t-1)}{2}(3 t+3)+s t(s+3 t+1)+s t(2 t+2 s)+s(2 t+2 s) \\
& M_{2}(H)=s t[(t+4)(t+5)+(t+5)(2 t+4)+(2 t+s+2)(t+5)+(3 t+3)(t+5)+(s-1)(2 t+4)(t+5) \\
& +((s-1)(t+4)(2 t+4)+(3 t+3)(t+4)+(2 t+s+2)(t+4)+5(t+4) \\
& +3(t+4)+5(t+5)+15+(3 t+3)(2 t+s+2) \\
& \cdot(s-1)(2 t+4)(3 t+3)+(s-1)(2 t+4)(2 t+s+2)+\frac{(t-1)(t-2)}{2}(2 t+4)(2 t+4) \\
& +12+20+(t+5)(4)+(4)(2 t+4)+(5)(2 t+4)+(s+3 t+1)(2 t+s+2)+\frac{(t-1)}{2} \\
& \cdot(2 t+s+2)^{2}+(3 s+t-1)(2 t+s+2)+(s-1)(2 t+2 s)(2 t+s+2)+(s+3 t+1) \\
& \left.\cdot(2 t+4)+(2 t+2 s)(2 t+4)+\frac{(t-1)}{2}(3 t+3)^{2}+(s+3 t+1)(3 t+3)+(2 t+2 s)(3 t+3)\right] \\
& +s[(s+3 t-1)(3 s+t-1)+(s-1)(2 t+2 s)(3 s+t-1)+(s-1)(2 t+2 s)(s+3 t+1) \\
& +(s-1)(2 t+2 s)(2 s+2 t)+\frac{(s-1)}{2}(3 s+t-1)(3 s+t-1)+(s-1)(2 t+2 s)(3 s+t-1) \\
& \left.+\frac{(s-1)(s-2)}{2}(2 s+2 t)(2 t+2 s)+(s+3 t+1)(2 s+2 t)\right]+(s-1)(2 t+2 s)(2 s+2 t), \\
& { }^{m} M_{2}(H)=s t\left[\frac{1}{(t+4)(t+5)}+\frac{1}{(t+5)(2 t+4)}+\frac{1}{(2 t+s+2)(t+5)}+\frac{1}{(3 t+3)(t+5)}+(s-1)\right. \\
& \cdot \frac{1}{(2 t+4)(t+5)}+(s-1) \frac{1}{(2 t+4)(t+4)}+\frac{1}{(3 t+3)(t+4)}+\frac{1}{(2 t+s+2)(t+4)}+\frac{1}{(5)(t+4)} \\
& +\frac{1}{(3)(t+4)}+\frac{1}{(5)(t+5)}+\frac{1}{(3)(5)}+\frac{1}{(3 t+3)(2 t+s+2)}+(s-1) \frac{1}{(2 t+4)(3 t+3)}+(s-1) \\
& \cdot \frac{1}{(2 t+4)(2 t+s+2)}+\frac{(t-1)(t-2)}{2} \frac{1}{(2 t+4)(2 t+4)}+\frac{1}{12}+\frac{1}{20}+\frac{1}{4(t+5)}+\frac{1}{4(2 t+4)} \\
& +\frac{1}{5(2 t+4)}+\frac{1}{(s+3 t+1)(2 t+s+2)}+\frac{(t-1)}{2} \frac{1}{(2 t+s+2)(2 t+s+2)}+\frac{1}{(2 s+2 t)(2 t+4)} \\
& +\frac{1}{(2 t+s+2)(3 s+t-1)}+(s-1) \frac{1}{(2 s+2 t)(2 t+s+2)}+\frac{1}{(s+3 t+1)(2 t+4)}+\frac{(t-1)}{2}
\end{aligned}
$$




$$
\begin{aligned}
& \left.\cdot \frac{1}{(3 t+3)(3 t+3)}+\frac{1}{(s+3 t+1)(3 t+3)}+\frac{1}{(2 s+2 t)(3 t+3)}\right]+s\left[(s-1) \frac{1}{(2 s+2 t)(3 s+t-1)}\right. \\
& +(s-1) \frac{1}{(2 s+2 t)(s+3 t+1)}+(s-1) \frac{1}{(2 s+2 t)(2 s+2 t)}+\frac{(s-1)}{2} \frac{1}{(3 s+t-1)(3 s+t-1)} \\
& +(s-1) \frac{1}{(3 s+t-1)(2 t+2 s)}+\frac{1}{(s+3 t-1)(s+3 t-1)}+\frac{(s-1)(s-2)}{2} \\
& \left.\cdot \frac{1}{(2 s+2 t)(2 s+2 t)}+\frac{1}{(2 s+2 t)(s+3 t+1)}\right]+(s-1) \frac{1}{(2 s+2 t)(2 t+2 s)}, \\
& R_{\alpha}(H)=s t\left[\frac{1}{(t+4)^{\alpha}(t+5)^{\alpha}}+\frac{1}{(t+5)^{\alpha}(2 t+4)^{\alpha}}+\frac{1}{(2 t+s+2)^{\alpha}(t+5)^{\alpha}}\right. \\
& +\frac{1}{(3 t+3)^{\alpha}(t+5)^{\alpha}}+(s-1) \frac{1}{(2 t+4)^{\alpha}(t+5)^{\alpha}}+(s-1) \frac{1}{(2 t+4)^{\alpha}(t+4)^{\alpha}} \\
& +\frac{1}{(3 t+3)^{\alpha}(t+4)^{\alpha}}+\frac{1}{(2 t+s+2)^{\alpha}(t+4)^{\alpha}}+\frac{1}{(5)^{\alpha}(t+4)^{\alpha}} \\
& +\frac{1}{(3)^{\alpha}(t+4)^{\alpha}}+\frac{1}{(5)^{\alpha}(t+5)^{\alpha}}+\frac{1}{(3)^{\alpha}(5)^{\alpha}} \\
& +\frac{1}{(3 t+3)^{\alpha}(2 t+s+2)^{\alpha}}+(s-1) \frac{1}{(2 t+4)^{\alpha}(3 t+3)^{\alpha}}+(s-1) \frac{1}{(2 t+4)^{\alpha}(2 t+s+2)^{\alpha}} \\
& +\frac{(t-1)(t-2)}{2} \frac{1}{(2 t+4)^{\alpha}(2 t+4)^{\alpha}}+\frac{1}{(3)^{\alpha}(4)^{\alpha}}+\frac{1}{(5)^{\alpha}(4)^{\alpha}}+\frac{1}{(t+5)^{\alpha}(4)^{\alpha}} \\
& +\frac{1}{(4)^{\alpha}(2 t+4)^{\alpha}}+\frac{1}{(5)^{\alpha}(2 t+4)^{\alpha}}+\frac{1}{(s+3 t+1)^{\alpha}(2 t+s+2)^{\alpha}} \\
& +\frac{(t-1)}{2} \frac{1}{(2 t+s+2)^{\alpha}(2 t+s+2)^{\alpha}}+\frac{1}{(2 t+s+2)^{\alpha}(3 s+t-1)^{\alpha}} \\
& +(s-1) \frac{1}{(2 s+2 t)^{\alpha}(2 t+s+2)^{\alpha}}+\frac{1}{(s+3 t+1)^{\alpha}(2 t+4)^{\alpha}}+\frac{1}{(2 s+2 t)^{\alpha}(2 t+4)^{\alpha}} \\
& \left.+\frac{(t-1)}{2} \frac{1}{(3 t+3)^{\alpha}(3 t+3)^{\alpha}}+\frac{1}{(s+3 t+1)^{\alpha}(3 t+3)^{\alpha}}+\frac{1}{(2 s+2 t)^{\alpha}(3 t+3)^{\alpha}}\right] \\
& +s\left[\frac{1}{(2 s+2 t)^{\alpha}(s+3 t+1)^{\alpha}}+(s-1) \frac{1}{(2 s+2 t)^{\alpha}(3 s+t-1)^{\alpha}}+(s-1)\right. \\
& \cdot \frac{1}{(2 s+2 t)^{\alpha}(s+3 t+1)^{\alpha}}+(s-1) \frac{1}{(2 s+2 t)^{\alpha}(2 s+2 t)^{\alpha}}+\frac{(s-1)}{2} \\
& \cdot \frac{1}{(3 s+t-1)^{\alpha}(3 s+t-1)^{\alpha}}+(s-1) \frac{1}{(3 s+t-1)^{\alpha}(2 s+2 t)^{\alpha}} \\
& \left.+\frac{(s-1)(s-2)}{2} \frac{1}{(2 s+2 t)^{\alpha}(2 s+2 t)^{\alpha}}+\frac{1}{(s+3 t-1)^{\alpha}(s+3 t-1)^{\alpha}}\right] \\
& +(s-1) \frac{1}{(2 s+2 t)^{\alpha}(2 s+2 t)^{\alpha}}, \\
& \mathrm{RR}_{\alpha}(H)=s t\left[(t+4)^{\alpha}(t+5)^{\alpha}+(t+5)(2 t+4)^{\alpha}+(2 t+s+2)(t+5)^{\alpha}+(3 t+3)^{\alpha}(t+5)^{\alpha}\right. \\
& +(s-1)(2 t+4)^{\alpha}(t+5)^{\alpha}+(s-1)(2 t+4)^{\alpha}(t+4)^{\alpha}+(3 t+3)^{\alpha}(t+4)^{\alpha}+(2 t+s+2)^{\alpha} \\
& \cdot(2 t+s+2)^{\alpha}+(5)^{\alpha}(t+4)^{\alpha}+(3)^{\alpha}(t+4)^{\alpha}+(5)^{\alpha}(t+5)^{\alpha}+(3)^{\alpha}(5)^{\alpha}+(3 t+3)^{\alpha} \\
& \cdot(2 t+s+2)^{\alpha}+(s-1)(2 t+4)^{\alpha}(3 t+3)^{\alpha}+(s-1)(2 t+4)^{\alpha}(2 t+s+2)^{\alpha} \\
& +\frac{(t-1)(t-2)}{2}(2 t+4)^{\alpha}(2 t+4)^{\alpha}+(3)^{\alpha}(4)^{\alpha}+(5)^{\alpha}(4)^{\alpha}+(t+5)^{\alpha}(4)^{\alpha}+(4)^{\alpha}(2 t+4)^{\alpha} \\
& +(5)^{\alpha}(2 t+4)^{\alpha}+(s+3 t+1)^{\alpha}(2 t+s+2)^{\alpha}+\frac{(t-1)}{2}(2 p+s+2)^{\alpha}(2 t+s+2)^{\alpha} \\
& +(2 t+s+2)^{\alpha}(3 s+t-1)^{\alpha}+(s-1)(2 t+2 s)^{\alpha}(2 t+s+2)^{\alpha}+(s+3 t+1)^{\alpha}(2 t+4)^{\alpha}
\end{aligned}
$$




$$
\begin{aligned}
& +(2 t+2 s)^{\alpha}(2 t+4)^{\alpha}+\frac{(t-1)}{2}(3 t+3)^{\alpha}(3 t+3)^{\alpha}+(s+3 t+1)^{\alpha}(3 t+3)^{\alpha} \\
& \left.+(2 t+2 s)^{\alpha}(3 t+3)^{\alpha}\right]+s\left[(s+3 t-1)^{\alpha}(3 s+t-1)^{\alpha}+\frac{(s-1)(s-2)}{2}\right. \\
& \cdot(2 t+2 s)^{\alpha}(2 s+2 t)^{\alpha}+(s-1)(2 t+2 s)^{\alpha}(3 s+t-1)^{\alpha}+(s-1)(2 t+2 s)^{\alpha} \\
& \cdot(s+3 t+1)^{\alpha}+(s-1)(2 t+2 s)^{\alpha}(2 s+2 t)^{\alpha}+\frac{(s-1)}{2}(3 s+t-1)^{\alpha}(3 s+t-1)^{\alpha} \\
& \left.+(s-1)(3 s+t-1)^{\alpha}(2 s+2 t)^{\alpha}+(2 t+2 s)^{\alpha}(s+3 t+1)^{\alpha}\right]+(s-1)(2 t+2 s)^{\alpha}(2 s+2 t)^{\alpha} \text {, } \\
& \operatorname{SDD}(H)=s t\left[\frac{(t+5)}{t+4}+\frac{(2 t+4)}{t+5}+\frac{(t+5)}{2 t+s+2}+\frac{(t+5)}{3 t+3}+(s-1) \frac{(t+5)}{2 t+4}+(s-1) \frac{(t+4)}{2 t+4}+\frac{(t+4)}{3 t+3}\right. \\
& +\frac{(t+4)}{2 t+s+2}+\frac{(t+4)}{5}+\frac{(t+4)}{3}+\frac{(t+5)}{5}+\frac{(5)}{3}+\frac{(2 t+s+2)}{3 t+3}+(s-1) \frac{(3 t+3)}{2 t+4}+(s-1) \\
& \frac{(2 t+s+2)}{2 t+4}+\frac{(t-1)(t-2)}{2}+\frac{(4)}{3}+\frac{4}{5}+\frac{4}{t+5}+\frac{(2 t+4)}{4}+\frac{(2 t+4)}{5}+\frac{(2 t+s+2)}{s+3 t+1} \\
& +\frac{(t-1)}{2} \frac{(2 t+s+2)}{2 t+s+2}+\frac{(3 s+t-1)}{2 t+s+2}+(s-1) \frac{(2 t+s+2)}{2 s+2 t}+\frac{(2 t+4)}{s+3 t+1}+\frac{(2 t+4)}{2 s+2 t} \\
& +\frac{(t-1)}{2}+\frac{(3 t+3)}{s+3 t+1}+\frac{(3 t+3)}{2 s+2 t}+(t+4) \frac{1}{t+5}+(t+5) \frac{1}{2 t+4}+(2 t+s+2) \frac{1}{t+5} \\
& +(3 t+3) \frac{1}{t+5}+(s-1)(2 t+4) \frac{1}{t+5}+(s+3 t+1) \frac{1}{2 t+4} \\
& +(s-1)(2 t+4) \frac{1}{t+4}+(3 t+3) \frac{1}{t+4}+(2 t+s+2) \frac{1}{t+4}+(5) \frac{1}{t+4}+(3) \frac{1}{t+4}+(5) \frac{1}{t+5} \\
& +(3) \frac{1}{5}+(3 t+3) \frac{1}{2 t+s+2}+(s-1)(2 t+4) \frac{1}{3 t+3}+(s-1)(2 t+4) \frac{1}{2 t+s+2}+\frac{(t-1)(t-2)}{2} \\
& \cdot(2 t+4) \frac{1}{2 t+4}+(3) \frac{1}{4}+(5) \frac{1}{4}+(t+5) \frac{1}{4}+(4) \frac{1}{2 t+4}+(5) \frac{1}{2 t+4}+(s+3 t+1) \frac{1}{2 t+s+2} \\
& +\frac{(t-1)}{2}(2 t+s+2) \frac{1}{2 t+s+2}+(2 t+s+2) \frac{1}{3 s+t-1}+(s-1)(2 t+2 s) \frac{1}{2 t+s+2} \\
& \left.\cdot \frac{1}{2 t+4}+(2 t+2 s) \frac{1}{2 t+4}+\frac{(t-1)}{2}(3 t+3) \frac{1}{3 t+3}+(s+3 t+1) \frac{1}{3 t+3}+(2 t+2 s) \frac{1}{3 t+3}\right] \\
& +s\left[\frac{(3 s+t-1)}{s+3 t-1}+\frac{(s-1)(s-2)}{2} \frac{(2 s+2 t)}{2 s+2 t}+(s-1) \frac{(3 s+t-1)}{2 s+2 t}+(s-1) \frac{(s+3 t+1)}{2 s+2 t}\right. \\
& +\frac{(s-1)}{2}+(s-1) \frac{(2 s+2 t)}{3 s+t-1}+\frac{(s+3 t+1)}{2 s+2 t}+1+\frac{(s-1)(s-2)}{2}+(s-1)(2 t+2 s) \\
& \frac{1}{3 s+t-1}+(s-1)(2 t+2 s) \frac{1}{s+3 t+1}+(s-1)(2 t+2 s) \frac{1}{2 s+2 t}+\frac{(s-1)}{2}+(s-1) \\
& \left.\cdot(3 s+t-1) \frac{1}{2 s+2 t}+(2 t+2 s) \frac{1}{s+3 t+1}\right]+(s-1)[2] \text {, } \\
& H(H)=2 s t\left[\frac{1}{2 t+9}+\frac{1}{3 t+9}+\frac{1}{3 t+s+7}+\frac{1}{4 t+8}+(s-1) \frac{1}{3 t+9}+(s-1) \frac{1}{3 t+8}+\frac{1}{4 t+7}\right. \\
& +\frac{1}{3 t+s+6}+\frac{1}{t+9}+\frac{1}{t+7}+\frac{1}{t+10}+\frac{1}{8}+\frac{1}{5 t+s+5}+(s-1) \frac{1}{5 t+7}+(s-1) \frac{1}{4 t+s+6} \\
& +\frac{(t-1)(t-2)}{2} \frac{1}{4 t+8}+\frac{1}{7}+\frac{1}{9}+\frac{1}{t+9}+\frac{1}{2 t+8}+\frac{1}{2 t+9}+\frac{1}{5 t+2 s+3}+\frac{(t-1)}{2} \frac{1}{4 t+2 s+4}
\end{aligned}
$$




$$
\begin{aligned}
& +\frac{1}{4 s+3 t+1}+(s-1) \frac{1}{4 t+3 s+2}+\frac{1}{s+5 t+5}+\frac{1}{2 s+4 t+4}+\frac{(t-1)}{2} \frac{1}{6 t+6}+\frac{1}{s+6 t+4} \\
& \left.+\frac{1}{5 t+2 s+3}\right]+2 s\left[\frac{1}{4 s+4 t-2}+\frac{(s-1)(s-2)}{2} \frac{1}{4 s+4 t}+(s-1) \frac{1}{5 s+3 t-1}+(s-1) \frac{1}{3 s+3 t+1}\right. \\
& \left.+(s-1) \frac{1}{4 s+4 t}+\frac{(s-1)}{2} \frac{1}{6 s+2 t-2}+(s-1) \frac{1}{3 t+5 s-1}+s \frac{1}{3 s+5 t+1}\right]+2(s-1) \frac{1}{4 s+4 t}, \\
& \operatorname{ISI}(H)=s t\left[\frac{(t+4)(t+5)}{2 t+9}+\frac{(t+5)(2 t+4)}{3 t+9}+\frac{(2 t+s+2)(2 t+s+2)}{3 t+s+7}+\frac{(3 t+3)(t+5)}{4 t+8}\right. \\
& +\frac{(s-1)(2 t+4)(t+5)}{3 t+9}+\frac{(s-1)(2 t+4)(t+4)}{3 t+8}+\frac{(3 t+3)(t+4)}{4 t+7}+\frac{(2 t+s+2)(t+4)}{3 t+s+6} \\
& +\frac{5(t+4)}{t+9}+\frac{3(t+4)}{t+7}+\frac{(5)(t+5)}{t+10}+\frac{15}{8}+\frac{12}{7}+\frac{(3 t+3)(2 t+s+2)}{5 t+s+5} \\
& +\frac{(s-1)(2 t+4)(3 t+3)}{5 t+7}+\frac{(s-1)(2 t+4)(2 t+s+2)}{4 t+s+6}+\frac{(t-1)(t-2)}{2} \frac{(2 t+4)(2 t+4)}{4 t+8} \\
& +\frac{20}{9}+\frac{(t+5)(4)}{t+9}+\frac{(4)(2 t+4)}{2 t+8}+\frac{(5)(2 t+4)}{2 t+9}+\frac{(s+3 t+1)(2 t+s+2)}{5 t+2 s+3} \\
& +\frac{(t-1)}{2} \frac{(2 t+s+2)(2 t+s+2)}{4 t+2 s+4}+\frac{(2 t+s+2)(3 s+t-1)}{4 s+3 t+1}+\frac{(s-1)(2 t+2 s)(2 t+s+2)}{3 t+3 s+2} \\
& +\frac{(s+3 t+1)(2 t+4)}{s+5 t+5}+\frac{(2 t+2 s)(2 t+4)}{4 t+2 s+4}+\frac{(t-1)}{2} \frac{(3 t+3)(3 t+3)}{6 t+6} \\
& \left.+\frac{(s+3 t+1)(3 t+3)}{s+6 t+4}+\frac{(2 t+2 s)(3 t+3)}{5 t+2 s+3}\right]+s\left[\frac{(2 t+2 s)(s+3 t+1)}{3 s+5 t+1}\right. \\
& +\frac{(s-1)(s-2)(2 s+2 t)(2 t+2 s)}{2(4 s+4 t)}+\frac{(s-1)(2 t+2 s)(3 s+t-1)}{5 s+3 t-1}+\frac{(s-1)(2 t+2 s)(s+3 t+1)}{3 s+5 t+1} \\
& +\frac{(s-1)(2 t+2 s)(2 s+2 t)}{4 s+4 t}+\frac{(s-1)(3 s+t-1)(3 s+t-1)}{2(6 s+2 t-2)}+\frac{(s-1)(3 s+t-1)(2 s+2 t)}{3 t+5 s+1} \\
& \left.+\frac{(s+3 t-1)(3 s+t-1)}{6 s+4 t-2}\right]+\frac{(s-1)(2 t+2 s)(2 s+2 t)}{4 s+4 t}, \\
& A(H)=s t\left[\frac{(t+4)^{3}(t+5)^{3}}{(2 t+7)^{3}}+\frac{(t+5)^{3}(2 t+4)^{3}}{(3 t+7)^{3}}+\frac{(2 t+s+2)^{3}(t+5)^{3}}{(3 t+s+5)^{3}}+\frac{(3 t+3)^{3}(t+5)^{3}}{(4 t+6)^{3}}+\frac{(3)^{3}(4)^{3}}{(5)^{3}}\right. \\
& +\frac{(s-1)(2 t+4)^{3}(t+5)^{3}}{(3 t+7)^{3}}+\frac{(s-1)(2 t+4)^{3}(t+4)^{3}}{(3 t+6)^{3}}+\frac{(3 t+3)^{3}(t+4)^{3}}{(4 t+5)^{3}}+\frac{(2 t+s+2)^{3}(2 t+s+2)^{3}}{(3 t+s+4)^{3}} \\
& +\frac{(5)^{3}(t+4)^{3}}{(t+7)^{3}}+\frac{(3)^{3}(t+4)^{3}}{(t+5)^{3}}+\frac{(5)^{3}(t+5)^{3}}{(t+8)^{3}}+\frac{(3)^{3}(5)^{3}}{(6)^{3}}+\frac{(3 t+3)^{3}(2 t+s+2)^{3}}{(5 t+s+3)^{3}}+\frac{(5)^{3}(2 t+4)^{3}}{(2 t+7)^{3}} \\
& +\frac{(s-1)(2 t+4)^{3}(3 t+3)^{3}}{(5 t+5)^{3}}+\frac{(s-1)(2 t+4)^{3}(2 t+s+2)^{3}}{(4 t+s+4)^{3}}+\frac{(2 t+4)^{3}(2 t+4)^{3}(t-1)(t-2)}{8(4 t+6)^{3}} \\
& +\frac{(5)^{3}(4)^{3}}{(7)^{3}}+\frac{(t+5)^{3}(4)^{3}}{(t+7)^{3}}+\frac{(4)^{3}(2 t+4)^{3}}{(2 t+6)^{3}}+\frac{(2 t+s+2)^{3}(2 t+s+2)^{3}(t-1)}{8(4 t+2 s+2)^{3}}+\frac{(2 t+2 s)^{3}(2 t+4)^{3}}{(2 s+4 t+2)^{3}} \\
& +\frac{(s+3 t+1)^{3}(2 t+s+2)^{3}}{(5 t+2 s+1)^{3}}+\frac{(2 t+s+2)^{3}(3 s+t-1)^{3}}{(4 s+3 t-1)^{3}}+\frac{(s-1)(2 t+2 s)^{3}(2 t+s+2)^{3}}{(4 t+3 s)^{3}} \\
& \left.+\frac{(s+3 t+1)^{3}(2 t+4)^{3}}{(s+5 t+3)^{3}}+\frac{(3 t+3)^{3}(3 t+3)^{3}(t-1)}{8(6 t+4)^{3}}+\frac{(s+3 t+1)^{3}(3 t+3)^{3}}{(s+6 t+2)^{3}}+\frac{(2 t+2 s)^{3}(3 t+3)^{3}}{(2 s+5 t+1)^{3}}\right]
\end{aligned}
$$




$$
\begin{aligned}
& +s\left[\frac{(2 t+2 s)^{3}(s+3 t+1)^{3}}{(3 s+5 t-1)^{3}}+\frac{(2 t+2 s)^{3}(2 s+2 t)^{3}(s-1)(s-2)}{8(4 s+4 t-2)^{3}}+\frac{(s-1)(2 t+2 s)^{3}(3 s+t-1)^{3}}{(5 s+3 t-5)^{3}}\right. \\
& +\frac{(s-1)(2 t+2 s)^{3}(s+3 t+1)^{3}}{(3 s+5 t-1)^{3}}+\frac{(3 s+t-1)^{3}(3 s+t-1)^{3}(s-1)}{8(6 s+2 t-4)^{3}}+\frac{(s-1)(3 s+t-1)^{3}(2 s+2 t)^{3}}{(3 t+5 s-3)^{3}} \\
& \left.+\frac{(s-1)(2 t+2 s)^{3}(2 s+2 t)^{3}}{(4 t+4 s-2)^{3}}+\frac{(s+3 t-1)^{3}(3 s+t-1)^{3}}{(4 s+4 t-2)^{3}}\right]+\frac{(s-1)(2 t+2 s)^{3}(2 s+2 t)^{3}}{(4 t+4 s-2)^{3}} .
\end{aligned}
$$

Proof. For line distribution shown in Table 2, use of formulas given in (19)-(28) will give the above desired expressions.

\section{Conclusion}

In this study, we computed different topological indices and polynomials for total graph and the line graph of total graph of the considered tree, i.e., Kragujevac tree. It would be fascinating in the future to discover the same outcomes for those chemical invariants that have not yet been evaluated.

\section{Data Availability}

The data used to support the findings of this study are cited at relevant places within the text as references.

\section{Conflicts of Interest}

The authors declare that they have no conflicts of interest.

\section{Authors' Contributions}

This work was equally contributed by all writers.

\section{References}

[1] S. A. Hosseini, M. B. Ahmed, and I. Gutman, "Kragujevac trees with minimal atom-bond connectivity index," MATCH Communications in Mathematical and in Computer Chemistry, vol. 71, pp. 5-20, 2014.

[2] B. Basavanagoud and S. Timmanaikar, "Computing first zagreb and forgotten indices of certain dominating transformation graphs of kragujevac trees," Journal of Computer and Mathematical Sciences, vol. 8, no. 3, pp. 50-61, 2017.

[3] M. Rezaei, M. S. Hosamani, M. R. Farahani, and M. K. Jamil, "On the terminal wiener index and Zagreb indices of Kragujevac tree," International Journal of Pure and Applied Mathematics, vol. 5, no. 113, pp. 617-625, 2017.

[4] B. Basavanagoud, "Computing certain topological indices of some dominating graph operations of Kragujevac Trees," Asian Journal of Mathematics and Computer Research, vol. 15, no. 1, pp. 30-40, 2017.

[5] B. Basavanagoud and S. Timmanaikar, "On the wiener and hyper wiener indices of certain Dominating Transformation Graphs of Kragujevac Trees," International Journal of Mathematical, vol. 8, no. 1, pp. 128-138, 2017.

[6] B. Basavanagoud and S. Timmanaikar, "Study on some topological indices of semientire and entire dominating graphs of kragujevac trees," Journal of Computer and Mathematical Sciences, vol. 9, no. 2, pp. 94-109, 2018.
[7] R. Cruz, J. Rada, and I. Gutman, "Topological indices of Kragujevac trees," Proyecciones (Antofagasta), vol. 33, no. 4, pp. 471-482, 2014.

[8] I. Gutman, N. Trinajstić, and C. F. Wilcox, "Graph theory and molecular orbitals. Total $\varphi$-electron energy of alternant hydrocarbons," Chemical Physics Letters, vol. 17, no. 4, pp. 535-538, 1972.

[9] M. H. Khalifeh, H. Yousefi-Azari, and A. R. Ashrafi, "The first and second Zagreb indices of some graph operations," Discrete Applied Mathematics, vol. 157, no. 4, pp. 804-811, 2009.

[10] H. Deng, D. Sarala, S. K. Ayyaswamy, and S. Balachandran, "The Zagreb indices of four operations on graphs," Applied Mathematics and Computation, vol. 275, pp. 422-431, 2016.

[11] A. E. Nabeel, "Zagreb polynomials of certain families of bendrimer nanostars," Tikrit Journal of Pure Science, vol. 20, no. $4,2015$.

[12] J. Hao, "Theorems about zagreb indices and modified zagreb indices," MATCH Communications in Mathematical and in Computer Chemistry, vol. 65, pp. 659-670, 2011.

[13] G. H. Shirdel, H. Rezapour, and A. M. Syadi, "The hyperZagreb index of graph operations," Iranian Journal of Mathematical Chemistry, vol. 4, no. 2, pp. 213-220, 2013.

[14] P. S. Ranjini, V. Lokesha, and I. N. Cangül, "On the zagreb indices of the line graphs of the subdivision graphs," Applied Mathematics and Computation, vol. 218, no. 3, pp. 699-702, 2011.

[15] B. Furtula and I. Gutman, "A forgotten topological index," Journal of Mathematical Chemistry, vol. 53, no. 4, pp. 1184-1190, 2015.

[16] I. Gutman, B. Furtula, and C. Elphick, "Three new/old vertexdegree based topological indices," MATCH Commun. Math. Comput. Chem, vol. 72, no. 24, pp. 617-632, 2014.

[17] V. R. Kulli, "The gourava indices and coindices of graphs," Annals of Pure and Applied Mathematics, vol. 14, no. 1, pp. 33-38, 2017.

[18] V. R. Kulli, "The product connectivity gourava index," Journal of Mathematical and Computational Science, vol. 8, no. 6, pp. 235-242, 2017.

[19] V. R. Kulli, "On the sum connectivity Gourava index," International Journal of Mathematical Archive, vol. 8, no. 6, pp. 211-217, 2017.

[20] V. R. Kulli, "On hyper-gourava indices and coindices," International Journal of Mathematical Archive, vol. 8, no. 12, pp. 116-120, 2017.

[21] M. S. Ahmad, W. Nazeer, S. M. Kang, and C. Y. Jung, " $M$-polynomials and degree based topological indices for the line graph of Firecracker graph," Global Journal of Pure and Applied Mathematics, vol. 13, no. 6, pp. 2749-2776, 2017.

[22] M. Eliasi and B. Taeri, "Four new sums of graphs and their wiener indices," Discrete Applied Mathematics, vol. 157, no. 4, pp. 794-803, 2009.

[23] M. R. Farahani, M. R. Jamil, M. K. Jamil, M. R. Rajesh Kanna, and S. M. Hosamani, "The wiener index and hasoya 
p0lynomial of the Subdivision Graph of Wheel $S\left(W_{n}\right)$ and the line graph subdivision of the wheel $L\left(S\left(W_{n}\right)\right)$," Applicationes Mathematicae, vol. 6, no. 2, pp. 21-24, 2016.

[24] M. R. Farahani, M. R. Jamil, and M. K. Jamil, "The shultz and modified schultz polynomials of the certain subdivision and line subdivision graphs," Journal of Chemical and Pharmaceutical Research, vol. 8, no. 3, pp. 51-57, 2016.

[25] M. Ajmal, W. Nazeer, W. Khalid, and S. M. Kang, "Forgotten polynomial and forgotten index for the line graphs of Banana tree graph, Firecracker graph and subdivision graphs," Global Journal of Pure and Applied Mathematics, vol. 13, no. 6, pp. 2673-2682, 2017.

[26] M. F. Nadeem, S. Zafar, and Z. Zahid, "On certain topological indices of the line graph of subdivision graphs," Applied Mathematics and Computation, vol. 271, pp. 790-794, 2015.

[27] Y. Gao, M. K. Jamil, A. Aslam, and M. R. Farahani, "Comutation of some new/old vertex-degree-based topological indices of line graph of subdivision graph of some nanosrtuctures," Journal of Optoelectronics and Biomedical Materials, vol. 9, no. 3, pp. 135-142, 2017.

[28] S. H. Bertz, "The bond graph," Journal of the Chemical Society, Chemical Communications, vol. 818-820, 1981.

[29] M. F. Nadeem, S. Zafar, and Z. Zahid, "On topological properties of the line graphs of subdivision graphs of certain nanostructures," Applied Mathematics and Computation, vol. 273, no. 273, pp. 125-130, 2016.

[30] W. Nazeer, M. Akmal, M. Imran, and M. R. Farahani, "On topological indices of line graph of subdivision of star and friendship graphs," International Journal of Pure and Applied Mathematics, vol. 118, no. 2, pp. 413-417, 2018. 